\title{
Propagation Effects in the Spin-Wave Spectrum of the Ferromagnetic Thin Film
}

\author{
Sławomir Mamica \\ Faculty of Physics, Adam Mickiewicz University in Poznań, Ulica Umultowska 85, 61-614 Poznań, Poland \\ Correspondence should be addressed to Sławomir Mamica; mamica@amu.edu.pl
}

Received 14 June 2015; Accepted 20 August 2015

Academic Editor: Victor V. Moshchalkov

Copyright ( 2015 Sławomir Mamica. This is an open access article distributed under the Creative Commons Attribution License, which permits unrestricted use, distribution, and reproduction in any medium, provided the original work is properly cited.

\begin{abstract}
Exchange spin waves propagating in magnetic thin films exhibit some dynamic effects, that is, the effects caused solely by the wave propagation. In this paper we put our attention in four phenomena of such kind: the surface (and subsurface) localization, the collapse of the bulk band into a single energy level, the reversal of the mode order in the spin-wave spectrum, and the dynamic separation of the thin film into two subsystems. We link these effects to properties of Hamiltonian matrix elements inherited from the spatial distribution of neighboring spins with respect to the film surface. We also provide necessary conditions for the occurrence of these effects.
\end{abstract}

\section{Introduction}

Magnetic thin films are the subject of intensive studies, both experimental [1-5] and theoretical [6-10], for more than half a century. This interest is stimulated by two main reasons: the huge progress in the fabrication technology of such structures [11] and the rich physics they exhibit. The latest is also inherited in more complicated systems such as magnetic multilayers [12-14] or thin-film magnonic crystals [15-18]. A particular class of phenomena is related to the parallel to the surface propagation of spin waves (in-plane propagation).

The propagation of spin waves in thin magnetic films leads to several effects including surface localization. For example, if magnetostatic interactions are taken into account famous Damon-Eshbach modes occur [19]. These modes are localized only at one surface, bottom or top, depending on the propagation direction. As a consequence nonreciprocity effect appears in the spin-wave spectrum for thin films with one surface differing from another [20]. Both effects are dynamic and disappear for standing spin waves, that is, for in-plane wave vector equal to zero. The dynamic localization appears also for purely exchange spin waves. Even in very simple model such as ferromagnetic thin film with natural surface and only nearest neighbor $(\mathrm{NN})$ exchange interactions taken into account the surface localization occurs for propagating spin waves $[21,22]$. In this case also another dynamic effect was observed, namely, the collapse of the bulk band, where all bulk modes are degenerated having the same energy [23].

Dynamic surface localization in thin magnetic films was studied theoretically in last decades [24-27] but it strongly gained in importance since spin polarized electron energy loss spectroscopy (SPEELS) was used to investigate propagating spin waves [28-30]. In contrast to the ferromagnetic resonance (FMR), which investigates standing spin waves, SPEELS gives possibility to scan the entire surface Brillouin zone (SBZ); thus it proves to be the powerful tool for the experimental study of propagation effects. The method allows experimental studies of the exchange interactions [31], magnetic anisotropy [32], or thermal properties [33]. For example, latest SPEELS researches show the interaction type in iron thin films to be strongly dependent on the substrate: from antiferromagnetic exchange [34] to DzyaloshinskiiMoriya one [35]. It allows also for the direct observation of spin waves localized dynamically at the surface [36].

In the planar model used in our work the thin film is treated as a set of layers representing lattice planes parallel to the surface. This allows us to reduce the problem to a onedimensional finite chain [7] with effective coupling between its elements (lattice planes). Propagation effects are strongly influenced by this effective interlayer coupling which reflects the total influence of exchange interactions from neighboring 
spins. The interlayer coupling was studied for standing spin waves in both dipolar and exchange coupled multilayers [37-42]. For exchange coupled layers the effective coupling depends on the wave vector of the propagating spin wave which may lead to its vanishing and the changing of its sign (between ferro- and antiferromagnetic) [43].

The spin-wave spectrum of different magnetic systems is strongly influenced by the range of considered interactions. For example, concurrence of the short range exchange and long range dipolar interactions generates menagerie of (meta)stable magnetic configurations [44-50] and causes rich spectrum of magnetic excitations [51-55] in magnetic dots, leads to the complete bandgap opening in magnonic crystals [56], or induces the spin-wave pinning in strongly curveted wires [57]. In the case of thin films it is responsible for the occurrence of negative group velocity [58] and the splitting of the spin-wave spectrum into subbands [14]. Also purely exchange spin waves exhibit new features if next nearest neighbors (NNN) interactions are considered, for example, subsurface localization [59] or separation of the film into subsystems [43].

These dynamic effects and their dependence on NNN interactions as well as on the crystallographic structure of thin film are the main objective of the present paper. We show that some of these effects could be useful for the determination of the NNN exchange interactions which is often a rather difficult task. One of more spectacular examples is the case of europium monochalcogenides (EuX). For EuS the literature data differ by the factor 2, and for EuO even the sign of $J_{N}$ remains in question [60-62]. The bulk band collapse, followed by the dynamic mode order reversal with changing wave vector, seems especially useful for this purpose.

In our work we use the planar model of the thin film with spin waves described by the Heisenberg Hamiltonian which contains NN and NNN interactions. The model has been known since the late sixties and its derivation is performed in several papers (see, e.g., [7]); thus we shall only recall its main assumptions in Section 2. In the next sections we use this model to explain dynamic (propagation) effects in the spin-wave spectrum of thin films. In Section 3 we consider a surface localization of spin waves caused solely by their propagation and provide necessary conditions for this type of localization to occur. In Section 4 we study the dynamic collapse of the bulk band caused by the vanishing of the effective coupling between adjacent layers. For certain in-plane wave vectors all bulk modes in the spin-wave spectrum have equal energy, which makes the whole bulk band collapse into one energy level. The effect is usually followed by the reversal of the order of modes in two regions of the SBZ. In Section 5 we show that the thin film can separate into subsystems in which spin waves propagate independently for wave vectors from the border of the SBZ. Major results are summarized in Section 6. The paper is completed with two appendixes. In Appendix A we provide the neighbors distribution in the thin films considered in the paper. Appendix B contains the explicit dependence on the in-plane wave vector of structural sums introduced in Section 2.

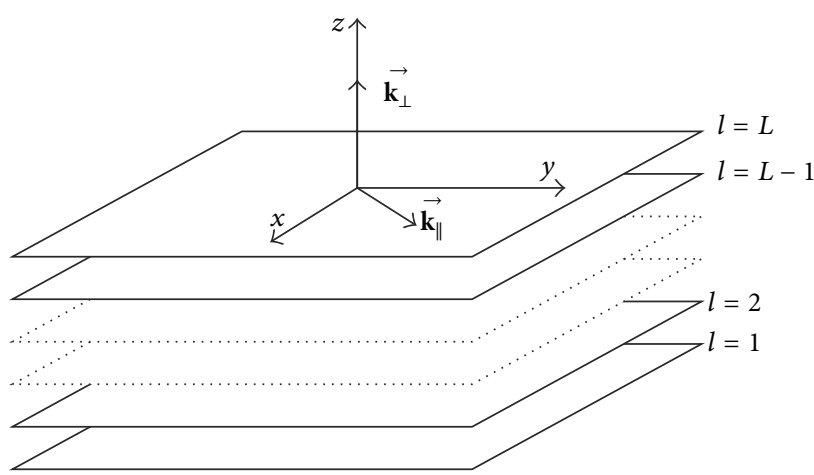

Figure 1: The planar model of thin film: the film can be treated as a set of $L$ crystallographic planes (layers) parallel to the surface. The wave vector is decomposed into two components, $\mathbf{k}_{\|}=\left(k_{x}, k_{y}\right)$ and $\mathbf{k}_{\perp}$, parallel and perpendicular to the surface, respectively.

\section{The Model}

The schematic plot of a model is shown in Figure 1. The system under consideration is a thin magnetic film that consists of spins $S$ arranged in sites of a crystal lattice. The film can be treated as a set of crystallographic planes (layers) parallel to its surface $(x-y$ plane). An external magnetic field strong enough to stabilize the homogenous ferromagnetic ground state is applied perpendicularly to the surface (along the $z$ direction). Under this assumption the Zeeman energy can be neglected as it only results in a uniform shift of the spin-wave spectrum. The wave vector can be decomposed into a parallel to the film surface in-plane component $\mathbf{k}_{\|}=\left(k_{x}, k_{y}\right)$ and perpendicular component $\mathbf{k}_{\perp}$. The first component is related to the in-plane propagation of spin waves while the second one describes their quantization along the film thickness.

Considering uniform NN and NNN exchange interactions the Hamiltonian of the film takes the following form:

$$
\widehat{\mathscr{H}}=-2 J \sum_{l \mathbf{j} ; l^{\prime} \mathbf{j}^{\prime}} \widehat{\mathbf{S}}_{l \mathbf{j}} \cdot \widehat{\mathbf{S}}_{l^{\prime} \mathbf{j}^{\prime}}-2 J_{N} \sum_{l \mathbf{j} ; l^{\prime} \mathbf{j}^{\prime}} \widehat{\mathbf{S}}_{l \mathbf{j}} \cdot \widehat{\mathbf{S}}_{l^{\prime} \mathbf{j}^{\prime}},
$$

where $J$ and $J_{N}$ are the NN and NNN exchange integrals, respectively. In the planar model of thin film the position of each spin is given by the layer number, $l$, and the position vector within the layer, $\mathbf{j}$. The symbols $l^{\prime} \mathbf{j}^{\prime}$ in (1) refer to the neighbors of the spin $l \mathbf{j}$, nearest in the first summation and next nearest in the second one. The spin from the plane $l$ can have neighbors in the same lattice plane $\left(\mathbf{j}^{\prime} \in l^{\prime}=l\right)$, in neighboring planes $\left(\mathbf{j}^{\prime} \in l^{\prime}=l+1\right)$, or in further planes.

The detailed description of the diagonalization procedure of Hamiltonian (1) is given in several papers (see, e.g., [7]). The first step is the Holstein-Primakoff transformation, that is, the mapping to boson operators. Then the Fourier transformation in the plane of the film is performed and, subsequently, the Bogolyubov-Tyablikov transformation in the direction perpendicular to it (mapping to the wave-vector space). The assumptions the method is based on make it valid for low concentration of the spin waves (low temperatures in the case of thermal magnons) and for thin films large in the directions parallel to the surface. 
The final outcome is an $L \times L$ band matrix, where $L$ is the number of layers forming the thin film, in the following form:

$$
\widehat{\mathscr{H}}=\left[\begin{array}{ccccccc}
R-a_{1} & C_{1} & C_{2} & & & & \\
C_{1} & R-a_{2} & C_{1} & C_{2} & & & \\
C_{2} & C_{1} & R & C_{1} & C_{2} & & \\
& \ddots & \ddots & \ddots & \ddots & \ddots & \\
& & C_{2} & C_{1} & R & C_{1} & C_{2} \\
& & & C_{2} & C_{1} & R-a_{2} & C_{1} \\
& & & & C_{2} & C_{1} & R-a_{1}
\end{array}\right] .
$$

The number of nonzero elements depends on the spatial distribution of the NN and NNN (provided in Appendix A) and their explicit forms depend on the exchange integrals $J$ and $J_{N}$, as well as on the in-plane wave vector.

Matrix (2) is typical for one-dimensional chain where the interaction between its links is described by the off-diagonal elements of the matrix. Other entries of the Hamiltonian matrix have the following meanings: $R$ represents the onsite energy; $a_{1-2}$ are surface (subsurface) parameters (see, e.g., [7] for more details). In other words, the method used transforms thin film into a one-dimensional chain, where the role of chain elements is played by lattice planes parallel to the surface. This means the off-diagonal entries of the matrix describe the effective coupling between lattice planes: $C_{1}$ refers to the interaction between nearest layers, $C_{2}$ to the interaction between next nearest ones, and so forth. In our microscopic model this effective interlayer coupling reflects the total influence of exchange interactions from neighboring spins taking into account NN and NNN. The coupling depends not only on exchange integrals and the surface cut (NN and NNN distribution) but also on the inplane wave vector which is the reason for the occurrence of propagation (dynamic) effects in the spin-wave spectrum. Thus the knowledge of the dependence of off-diagonal elements on the in-plane wave vector allows forecasting the propagation effects. It is also worth noticing that the Zeeman energy, as an on-site energy, only affects the diagonal terms, shifting the whole spectrum of eigenvalues in a uniform way.

During the diagonalization of the Hamiltonian it is useful to introduce the so-called structural sums, which describe the spatial distribution of the NN and NNN [7], defined as follows:

$$
\Gamma_{n}=\sum_{\mathbf{j}^{\prime}} \exp \left( \pm i \mathbf{k}_{\|} \cdot\left(\mathbf{j}-\mathbf{j}^{\prime}\right)\right), \quad\left(\mathbf{j} \in \mathbf{l}, \mathbf{j}^{\prime} \in l^{\prime}=l+n\right)
$$

where summation over $\mathbf{j}^{\prime}$ runs over the projections of (next) nearest neighbors of the spin in the plane $l$ belonging to the plane $l+n$. The explicit forms of structural sums for surface cuts considered in this paper are given in Appendix B.
Elements of the Hamiltonian matrix expressed in terms of structural sums are the following:

$$
\begin{aligned}
R= & 4 S \sum_{i=1}^{2}\left(J z_{i}+J_{N} z_{i}^{N}\right) \\
& -2 S\left(J\left(\Gamma_{0}-z_{0}\right)+J_{N}\left(\Gamma_{0}^{N}-z_{0}^{N}\right)\right), \\
C_{n}= & -2 S\left(J \Gamma_{n}+J_{N} \Gamma_{n}^{N}\right) \\
a_{n}= & 2 S \sum_{i=n}^{2}\left(J z_{i}+J_{N} z_{i}^{N}\right)
\end{aligned}
$$

where $n=1,2$. Please notice that dependence of Hamiltonian matrix elements on the in-plane wave vector is introduced only by structural sums. Therefore the evolution of these sums with $\mathbf{k}_{\|}$determines propagation effects.

\section{Dynamic Surface Localization}

Surface localization of spin waves in thin magnetic films is widely studied in literature both experimentally and theoretically. For exchange spin waves it was found that the key factor for the surface localization is geometric disposition of interacting neighbors with respect to the surface rather than the interaction range: the emergence of surface states is due to interactions with neighbors the position vectors of which are oblique to the film surface, irrespectively of whether these "oblique" interactions are NN or NNN type [21, 22, 63]. Here we systemize the results and link them to the properties of Hamiltonian matrix elements. We also show that the range of interactions is important as well, however not the direct spinspin interactions but the effective interlayer ones.

The geometric disposition of neighboring spins is particularly important in the case of purely dynamical localization, that is, for the thin film with natural surfaces (no pinning). In such system there is no surface localization for the SBZ center but it could occur for nonzero in-plane wave vectors (propagating spin waves). To obtain surface localization for $\mathbf{k}_{\|}=0$ (which can be detected by FMR experiments) one needs to introduce surface anisotropy to generate additional pinning at the surface. The anisotropy has large influence on the surface localization and can cover the dynamical localization effect. Therefore it is not taken into account in this paper. (The influence of the anisotropy on the spin-wave localization is studied, e.g., in [64].)

The simplest case is $\mathrm{sc}(001)$ surface cut for which the Hamiltonian matrix is three-diagonal only. This is a consequence of the NN and NNN disposition. Every spin has $4 \mathrm{NN}$ and $4 \mathrm{NNN}$ at the same lattice plane; at the adjacent plane it has $1 \mathrm{NN}$ and $4 \mathrm{NNN}$ (see Appendix A). This means the effective coupling is restricted only to the neighboring lattice planes even if we include NNN interactions. In such case, that 
is, if the Hamiltonian matrix is three-diagonal, conditions for surface localization are [7]

$$
\begin{aligned}
\left|\frac{C}{a}\right| & <1, \\
\frac{|C / a|(L-1)}{L+1} & <1,
\end{aligned}
$$

for symmetric and antisymmetric mode, respectively. Here $C$ is off-diagonal element and $a$ is the surface parameter. Please notice that the surface parameter according to (6) depends only on the number of NN and NNN at the adjacent plane so the dynamic surface localization can be induced solely by $C$ through its dependence on structural sums (and then on in-plane wave vector).

According to the definition (3) if the position vector of the neighboring spin from adjacent layer is perpendicular to the surface (we will call it perpendicular neighbor) then the corresponding structural sum is equal to 1 (the number of adequate neighbors) independently of $\mathbf{k}_{\|}$. In this case $C=-a$ and neither condition (7) nor (8) is fulfilled and thus surface states cannot occur. In a consequence the necessary condition for the dynamic localization is an oblique disposition of the neighbors from adjacent lattice plane, which makes offdiagonal elements of the Hamiltonian matrix functions of the in-plane wave vector. For example, for sc(001) surface cut there is only one "perpendicular" nearest neighbor at the layer $l+1$ and thus corresponding structural sum $\Gamma_{1}=1$. The structural sum for NNN is given by $\Gamma_{1}^{N}=2\left(\cos k_{x}+\cos k_{y}\right)$ which means these 4 "oblique" neighbors form layer $l+1$ are responsible for eventual surface localization. Thus, the offdiagonal element $C$ is given by

$$
C=-2 S\left(J+2 J_{N}\left(\cos k_{x}+\cos k_{y}\right)\right),
$$

where $k_{x}$ and $k_{y}$ are components of the in-plane wave vector $\mathbf{k}_{\|}$.

In Figure 2 we show exemplary spin-wave spectra for sc(001) thin films consisting of $L=7$ lattice planes. The spectra are plotted along the high-symmetry path marked as a green solid line in the surface Brillouin zone (SBZ) shown in the inset in Figure 2(a). In the first case NNN interactions are assumed to be ferromagnetic (FM) with the ratio of NNN to NN exchange integral set to $J_{N} / J=0.5$ (Figure 2(a)). The second spectrum (Figure 2(b)) is obtained for antiferromagnetic (AFM) NNN interactions with $J_{N} / J=$ -0.5 . In both graphs the shaded area represents the bulk band. Below each spectrum we provide a graph with $C\left(\mathbf{k}_{\|}\right)$ plotted as a green dotted line accompanied with horizontal blue lines for $\pm a$ (dashed lines) and $\pm a(L-1) /(L+1)$ (solid lines). In Figures 2(c)-2(h) we show spin-wave profiles for some particular wave vectors marked with purple lines in the corresponding spectra.

For $J_{N} / J=0.5$ case surface parameter $a=6$ and condition (7) means that $|C|<6\left(C\left(\mathbf{k}_{\|}\right)\right.$should be between dashed lines in Figure 2(a)) which is fulfilled for any $\mathbf{k}_{\|} \neq 0$; that is, there is a symmetric surface state within the whole SBZ except point $\Gamma$. In Figure 2(c) we show spin-wave profiles for the wave vector between points $\Gamma$ and $\Delta$ in the SBZ. The lowest mode is localized at both surfaces while all other modes are bulk modes. The second surface mode, antisymmetric, occurs for wave vector large enough to fulfill condition (8) which in the case in question means $|C|<4.5\left(C\left(\mathbf{k}_{\|}\right)\right.$between solid lines in Figure 2(a)). The points at which this mode becomes localized are marked by red arrows in Figure 2(a). In Figure 2(d) we show spin-wave profiles for point $X$ in the SBZ where two lowest modes are localized. (The third profile given in panel (e) shows the mode order reversal whose effect is explained in Section 4.)

For $J_{N} / J=-0.5$ case $a=-2$ and the surface localization of the symmetric mode appears in some region in the SBZ around its center (again, except point $\Gamma$ ), that is, if $C\left(\mathbf{k}_{\|}\right)$is between dashed lines in Figure 2(b). For larger $\mathbf{k}_{\|}$ there is no surface state in the spectrum. Condition (6) for antisymmetric mode localization boils down to $|C|<1.5$ $\left(C\left(\mathbf{k}_{\|}\right)\right.$between solid lines in Figure 2(b)) which is fulfilled for wave vectors from some ring around point $\Gamma$ (between points marked by red arrows, cf. profiles in Figures 2(f) and 2(g)).

Other surface cuts for which the Hamiltonian matrix is three-diagonal are bcc(110) and fcc(111) but now neighbors of both types, NN and NNN, which are located on the adjacent layer are disposed obliquely with respect to the surface plane. Therefore, both interactions are responsible for the dynamic surface localization.

Concerning the influence of the NN and NNN on the surface localization very instructive are surface cuts bcc $(001)$ and fcc (001). In both cases there are $4 \mathrm{NN}$ located "obliquely" at the adjacent plane $(l+1)$ and one "perpendicular" NNN at next-neighboring plane $(l+2)$. This means only $\mathrm{NN}$ are responsible for spin-wave localization. In other words, for the hypothetical situation when NN interactions are switched off $(J=0)$ and only NNN interactions are taken into account $\left(J_{N} \neq 0\right)$ there will be no surface states in the spinwave spectrum. Exemplary spectra are shown in Figure 3 for $\mathrm{bcc}(001)$ thin film consisting of 11 lattice planes $(L=11)$ with only one type of interactions taken into account: $\mathrm{NN}$ in panel (a) and NNN in panel (b). For the first case $a_{1}=8$ and the localization condition for symmetric mode is fulfilled for any in-plane wave vector except $0\left(C_{1}\left(\mathbf{k}_{\|}\right)\right.$above dashed blue line in Figure 3(a)). For antisymmetric mode the condition is $\left|C_{1}\right|<20 / 3=6.67$; thus the second surface state occurs if $C_{1}\left(\mathbf{k}_{\|}\right)$is above solid blue line (between points marked by red arrows). In Figure 3(b), that is, for the hypothetical case of only NNN interactions switched on, there is no surface localization due to the perpendicular disposition of the $\mathrm{NNN}$ from the plane $l+2$.

In the very last case the Hamiltonian matrix is fivediagonal; thus we should take into account both surface parameters $a_{1}$ and $a_{2}$, and simple conditions (7) and (8) for the surface localization are not valid anymore. This also means that the effective coupling between layers now extends up to the second-neighboring layer which is described by offdiagonal element $C_{2}$. For bcc(001) surface cut $C_{2}$ does not depend on $\mathbf{k}_{\|}$because at the layer $l+2$ there is only one "perpendicular" neighbor. Different situation holds for fcc(110) surface cut for which there are two "oblique" NNN at the layer $l+2$ (and four "oblique" $\mathrm{NN}$ at $l+1$ ). Hence, we expect surface localization caused by $\mathrm{NN}$ and additional localization 


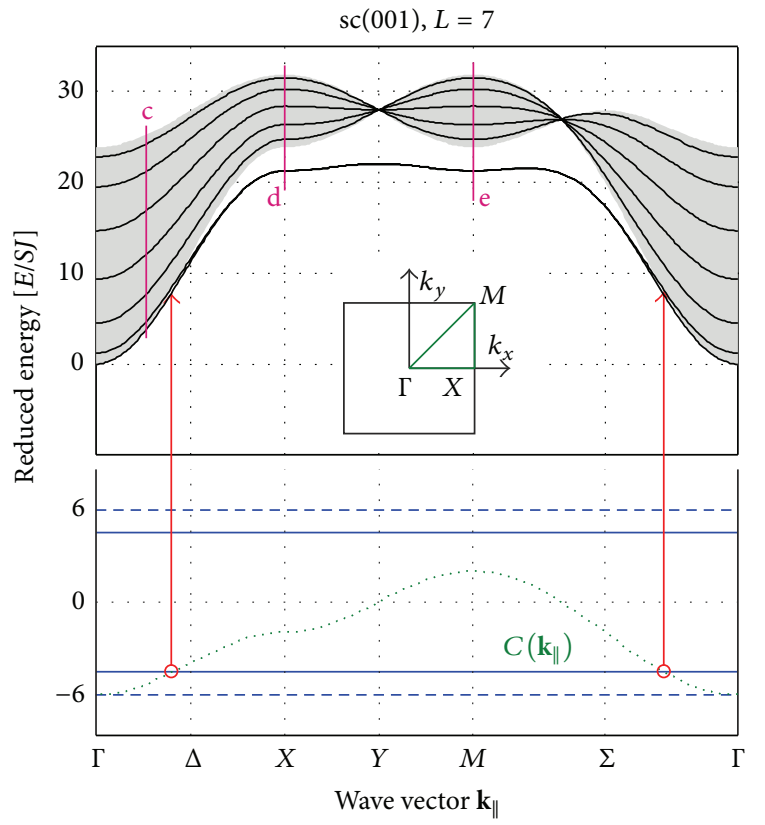

(a) $J_{N} / J=0.5$

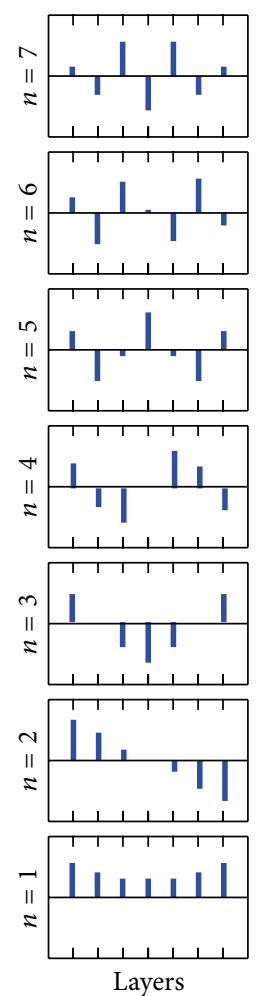

(c)

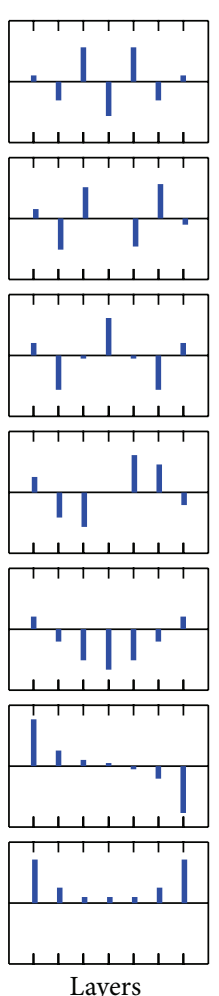

(d)

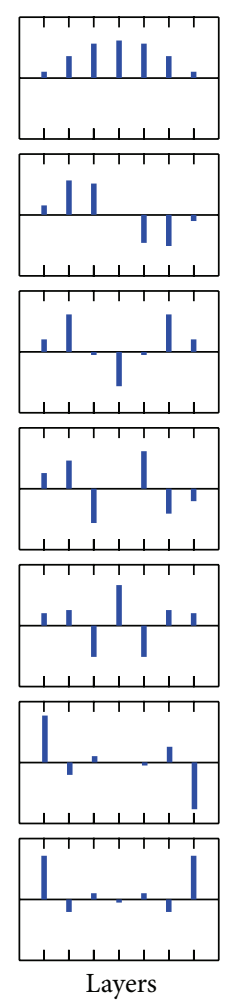

(e)

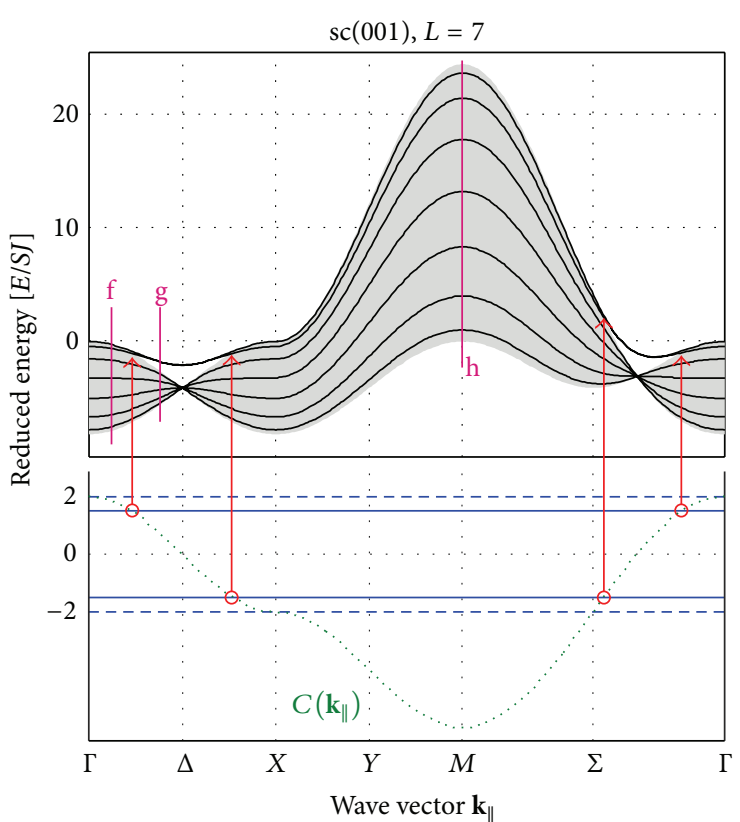

(b) $J_{N} / J=-0.5$
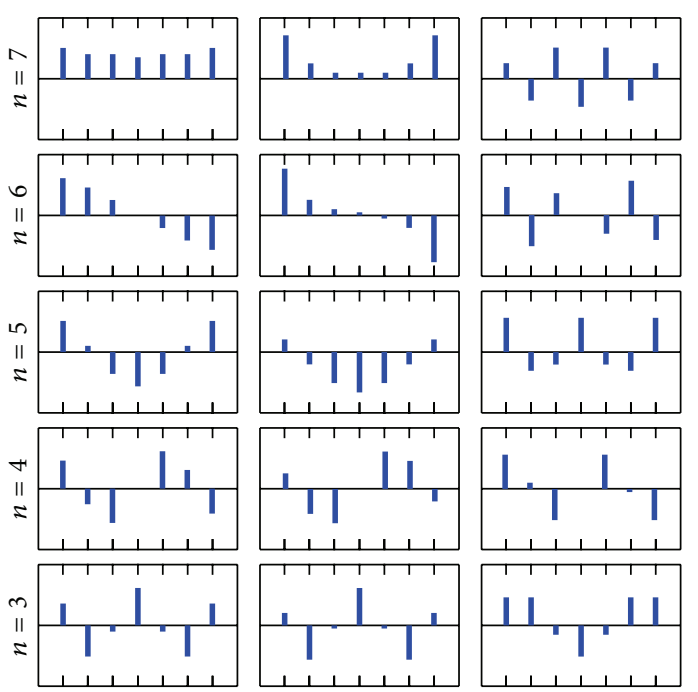

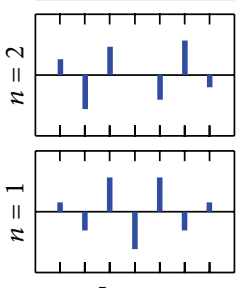

Layers

(f)

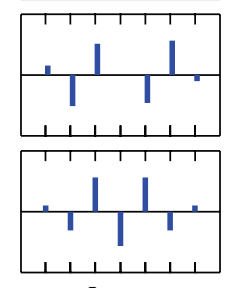

Layers

(g)

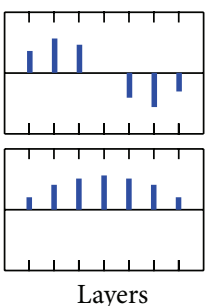

(h)

Figure 2: ((a), (b)) Spin-wave spectra of a 7-layer thick sc(001) thin film with NNN exchange interactions (a) ferromagnetic and (b) antiferromagnetic. Solid lines represent the energy of individual modes; the shaded area is the bulk band for in-plane spin waves. Each spectrum is plotted along the high-symmetry path shown in (a), inset. Below each spectrum the evolution of the off-diagonal Hamiltonian matrix element $C$ is shown as a green dotted line. Blue lines represent conditions for the surface localization of symmetric (solid lines) and antisymmetric (dashed lines) modes. Wave vectors for which the antisymmetric mode starts to localize are marked with red arrows. ((c)-(h)) Mode profiles corresponding to points in the SBZ marked with purple lines in ((a), (b)). 


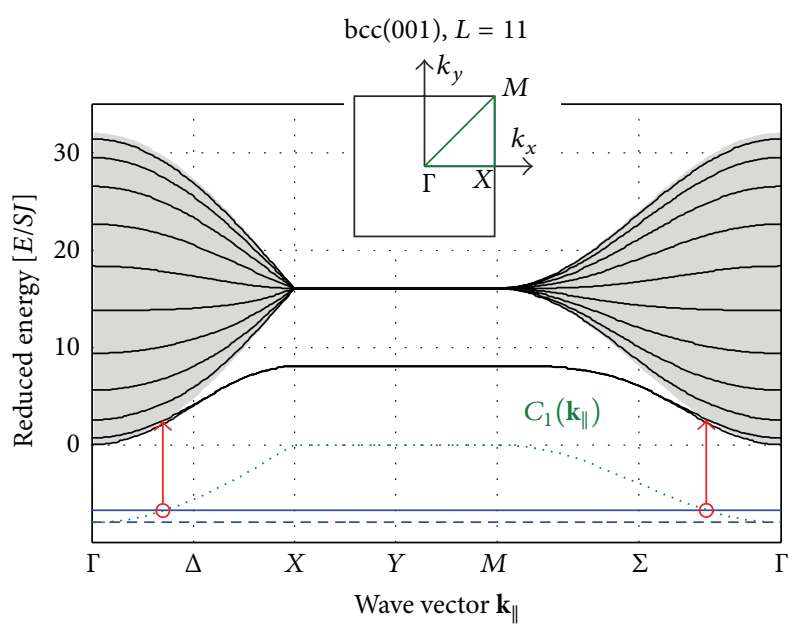

(a) $J \neq 0, J_{N}=0$

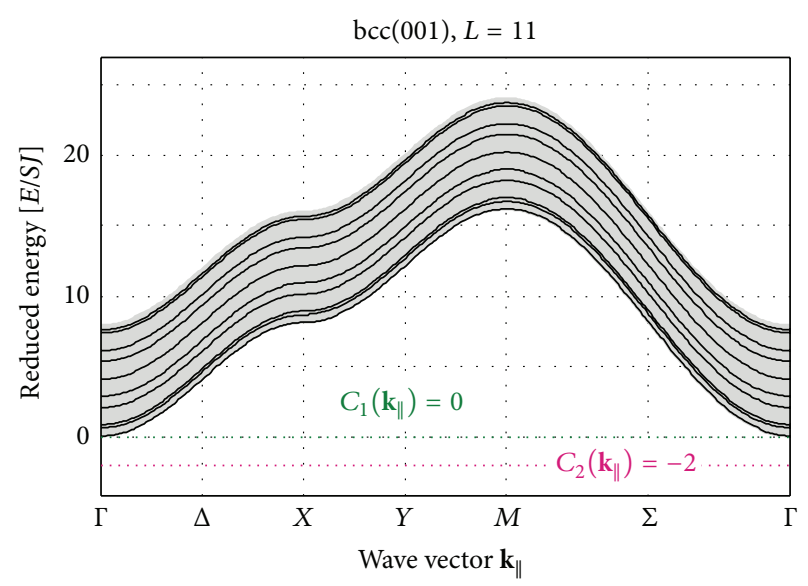

(b) $J_{N} \neq 0, J=0$

FIGURE 3: (a) Spin-wave spectrum of an 11-layer thick bcc(001) thin film with no NNN exchange interactions. Solid lines represent the energy of individual modes; the shaded area is the bulk band for in-plane spin waves. The spectrum is plotted along the high-symmetry path shown in an inset. The evolution of the off-diagonal Hamiltonian matrix element $C_{1}$ is shown as a green dotted line. Blue lines represent conditions for the surface localization of symmetric (solid line) and antisymmetric (dashed line) modes. Wave vectors for which the antisymmetric mode starts to localize are marked with red arrows. (b) The same as in (a) but for the hypothetical situation in which NN interactions are switched off while NNN interactions are switched on. The purple dotted line represents the evolution of the off-diagonal Hamiltonian matrix element $C_{2}$.

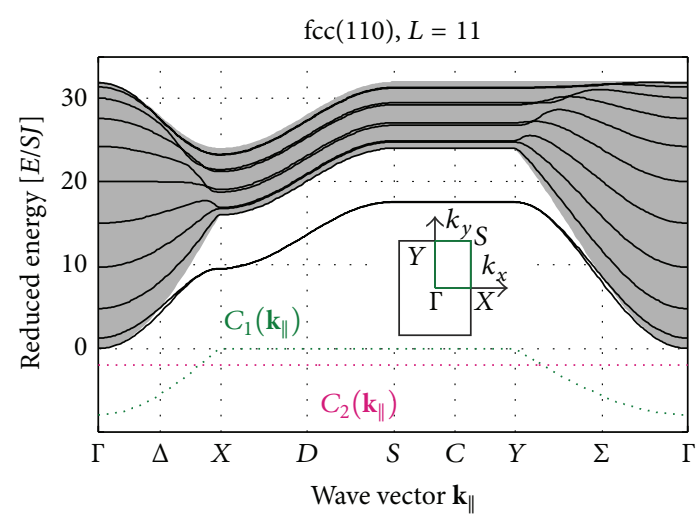

(a) $J_{N} / J=0$

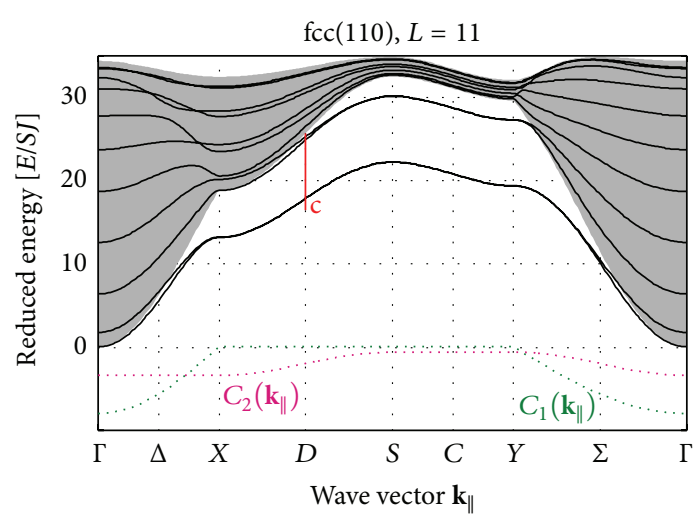

(b) $J_{N} / J=0.35$
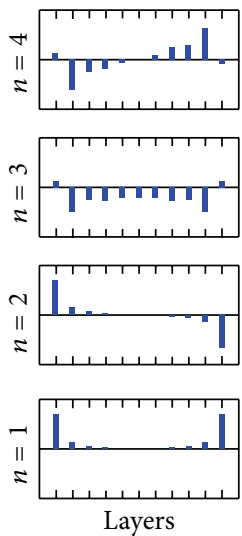

(c) $J_{N} / J=0.35$

FIGURE 4: ((a), (b)) Spin-wave spectra of an 11-layer thick fcc(110) thin film (a) without and (b) with NNN exchange interactions. Solid lines represent the energy of individual modes; the shaded area is the bulk band for in-plane spin waves. Each spectrum is plotted along the highsymmetry path shown in (a), inset, and accompanied with the evolution of off-diagonal elements $C_{1}$ (green dotted line) and $C_{2}$ (purple dotted line) of the Hamiltonian matrix. (c) Mode profiles of the four lowest modes corresponding to the point $D$ in the SBZ, marked with a red line in (b).

at one layer below surface (subsurface localization) caused by NNN. In Figure 4 we show two spin-wave spectra for fcc(110) thin film consisting of 11 layers without (panel (a)) and with (panel (b)) NNN interactions. In the first case (Figure 4(a)) there are two surface states emerging from the bulk band in some region of the SBZ (please notice that even if NNN interactions are switched off the matrix is five-diagonal and conditions (7) and (8) are not valid). If NNN interactions are taken into account (Figure 4(b)) two other modes go out of the bulk band. The profiles of the lowest modes are shown in Figure $4(\mathrm{c})$ : two lowest modes $(n=1,2)$ are localized at the surface while the next two modes $(n=3,4)$ are localized at the first plane below the surface layer. This subsurface localization is induced by the spin-wave propagation due to the oblique disposition of NNN from the layer $l+2$.

It is also worth noticing that each surface mode is localized at both surfaces. Moreover, in the case of symmetric thin film studied here the localization of particular mode is the same at both surfaces (the spin-wave profile is symmetric or antisymmetric). To differ one surface from the other one should introduce additional pinning. It could be done by taking into account the surface anisotropy [64]. However, 


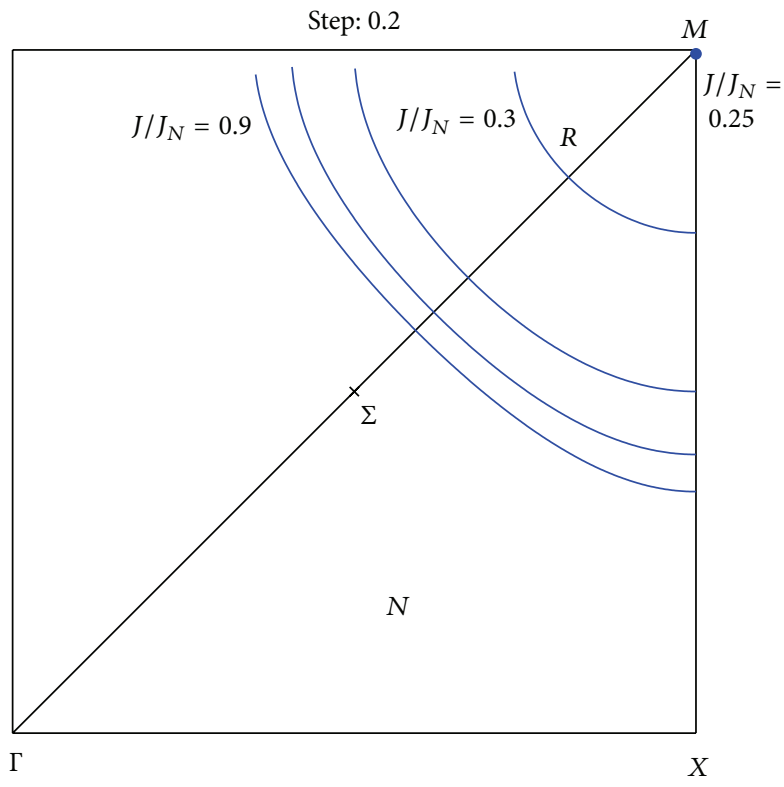

(a)

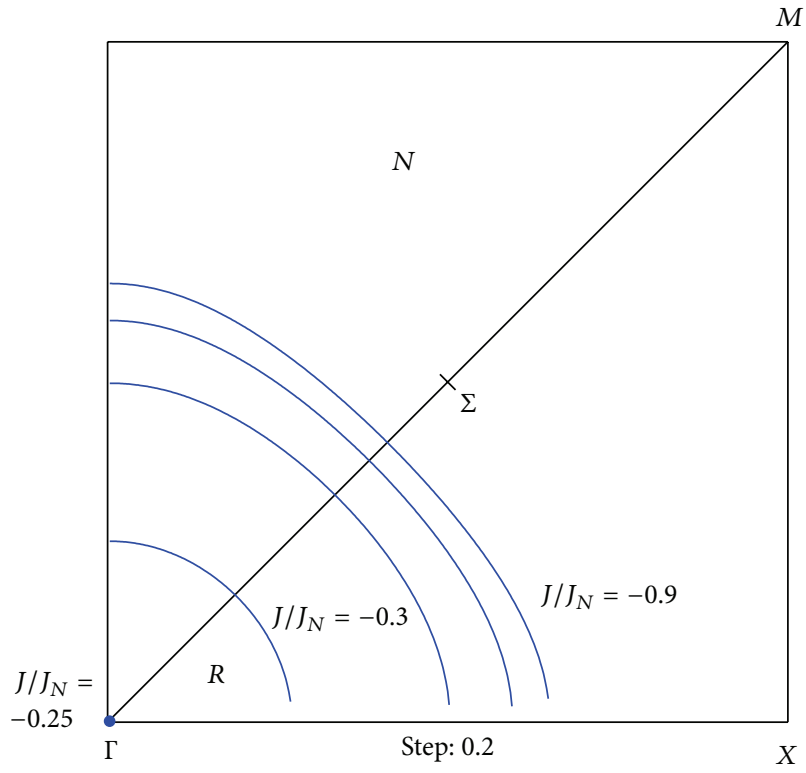

(b)

FIGURE 5: Two regions in the SBZ for sc(001) surface cut, with normal $(N)$ and reverse $(R)$ mode order in the spin-wave spectrum, for (a) ferromagnetic and (b) antiferromagnetic NNN interactions. The boundary between the regions shifts with growing $\left|J_{N}\right| / J$ towards the SBZ center as shown by blue curves. For the critical value $\left|J_{N}\right| / J=0.25$ the reversal domain does not occur (its boundary amounts to points $M$ and $\Gamma$ in (a) and (b), resp.).

anisotropy terms in the elements of the Hamiltonian matrix do not depend on the wave vector (see (19) and (20) in [64]). Therefore we do not observe such effects like nonreciprocity found in the case of Damon-Eshbach modes [20].

\section{Band Collapse and Mode Order Reversal}

Let us go back to the case for which the Hamiltonian matrix is three-diagonal only. Since off-diagonal element $C$ describes the effective coupling between neighboring layers only nearest layers are coupled. For $C<0$ the coupling is of ferromagnetic nature, that is, it favors the in-phase precession of spins belonging to neighboring lattice planes. By analogy with phonons [65], this type of excitation is referred to as acoustic. On the other hand, if $C>0$ the coupling has antiferromagnetic nature energetically favoring antiphase precession (if the external field saturates the sample; if not the situation is opposite). This type of excitation is called optic. In the case of phonons the acoustic mode has a lower energy than the optic mode and the same pattern is considered the normal order of modes in the spin-wave spectrum. If the energy of the optic mode is lower than that of the acoustic one, the spin-wave spectrum has a reversed mode order, which occurs as a consequence of AFM interaction between adjacent layers. (This problem is widely discussed for standing spin waves for both types of interlayer coupling, exchange and dipolar; see, e.g., [37, 39-42].) However, for propagating spin waves $C$ depends on in-plane wave vector which implies evolution of effective coupling within the SBZ. This means the character of effective coupling may change from FM to AFM for different wave vectors and,

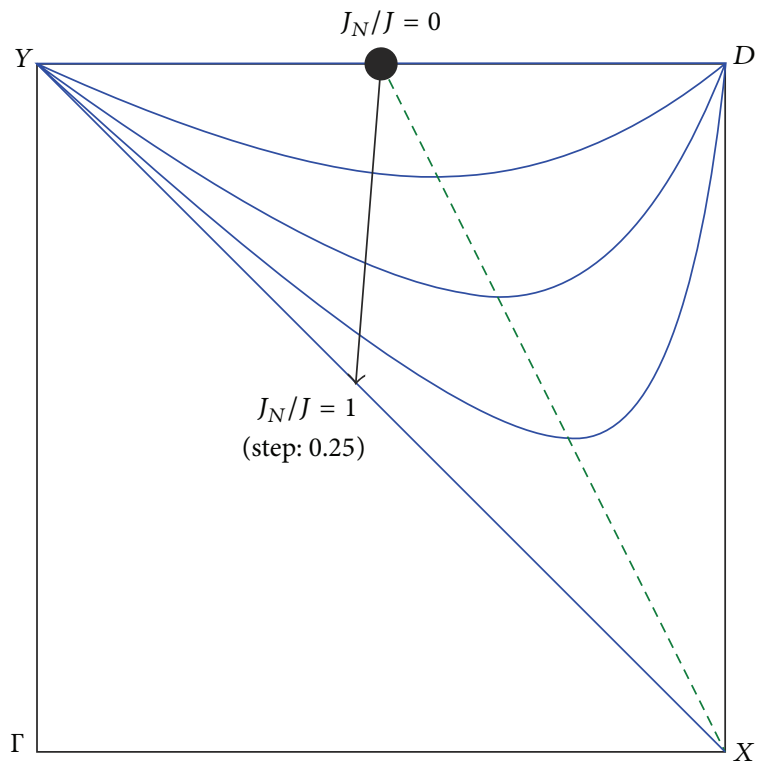

Figure 6: Two regions in the uniform zone for bcc(110) surface cut and ferromagnetic NNN interactions, with normal $(N)$ and reverse $(R)$ mode order in the spin-wave spectrum. The boundary between the regions shifts with growing $\left|J_{N}\right| / J$ as shown by the arrow. For no NNN interactions $\left(\left|J_{N}\right| / J=0\right)$ the reversal domain does not occur (its boundary amounts to the segment $D-Y$ ).

consequently, the order of modes in the spin-wave spectrum may change as well.

Such dynamic (due to the wave propagation) mode order reversal we observe in spin-wave spectra shown in Figure 2, that is, for $\mathrm{sc}(001)$ thin film composed of $L=7$ layers. 


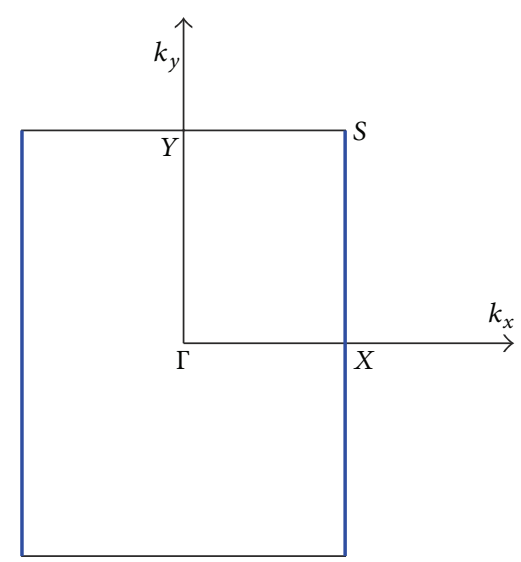

(a) $\operatorname{sc}(110)$

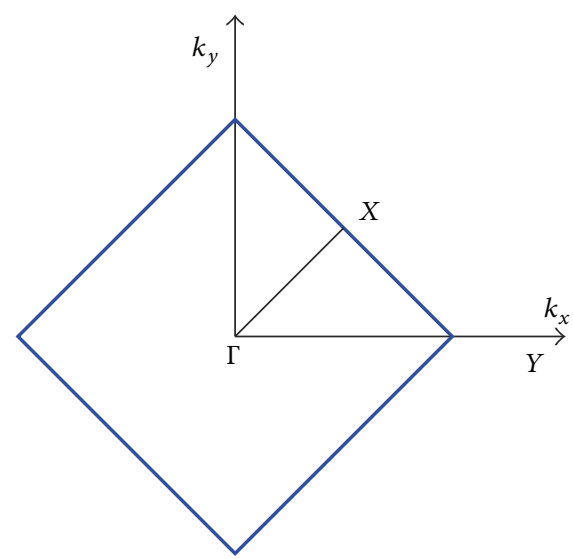

(b) fcc(001)

FIGURE 7: SBZ for (a) sc(110) and (b) fcc(001) surface cuts with the segments of the SBZ border at which the collapse of the bulk band occurs marked with bold blue lines.

In Figure 2(a) we show the spectrum for FM NNN interactions $\left(J_{N} / J=0.5\right)$ accompanied by the dependence of $C\left(\mathbf{k}_{\|}\right)$. Going along high-symmetry path in the SBZ (green line in the inset in Figure 2(a)) at point $\Gamma$ the effective interlayer coupling is FM $(C<0)$ until point $Y$ is reached. In Figures 2(c) and 2(d) we show spin-wave profiles for two points from this section of the high-symmetry path marked in panel (a) with purple lines. For both of them we observe normal order of modes with lowest mode acoustic and highest one optic. After crossing point $Y$ off-diagonal element $C$ becomes greater than zero and effective coupling is AFM. This results in reversed mode order shown in Figure 2(e) where spinwave profiles for point $M$ in the SBZ are plotted. Going further along the high-symmetry path $C$ restores its negative value after some point between $M$ and $\Sigma$. In other words, the reversed mode order occurs in some region of SBZ around point $M$.

A similar feature we observe for AFM NNN interactions for which case the spin-wave spectrum is presented in Figure 2(b). The main difference is that reversed mode order appears around point $\Gamma$ now (see the profiles provided in Figures 2(f) and $2(\mathrm{~g})$ ) because in this region $C>0$. After crossing point $X$ effective coupling is FM $(C<0)$ and the order of modes is normal (see spin-wave profiles for point $M$ in Figure 2(h)).

These two regions, of normal and reversed order of modes, are separated by line at which $C=0$ (see $C\left(\mathbf{k}_{\|}\right)$ dependence on Figures 2(a) and 2(b)). In such situation Hamiltonian matrix has nonzero elements only at its diagonal. In a consequence there are $L-2$ eigenvalues equal to $R$ (bulk modes) and two eigenvalues equal to $R-a$ (surface modes). This is what we call band collapse: whole bulk band is collapsed into one energy level. The collapse was discovered by Lévy et al. [23] in a semi-infinite ferromagnet of sc structure and (001) surface. Originally this effect was described as caused by NNN interactions. In [66] we studied this effect in thin films with different surface cuts and found it to occur even without NNN interactions. In [43] we show that the collapse may be followed by the reversal of the order of modes in the spin-wave spectrum.

Since $C$ depends on exchange integrals for NN and NNN also two effects described above should. For sc(001) surface cut element $C$ is given by (9) and the equation $C=0$ has real solutions only if $\left|J_{N} / J\right| \geq 0.25$. Therefore, if NNN interactions are too weak there is no bulk band collapse nor dynamical change of the mode order in the spin-wave spectrum of sc(001) films. For weak FM NNN interactions $C$ is negative and the order of modes in the spectrum is normal within the whole SBZ. On the other hand, for weak AFM NNN interactions the situation is opposite: $C>0$ and the order of modes is reversed for any $\mathbf{k}_{\|}$. For stronger NNN interactions the solutions for different $J_{N} / J$ ratios are plotted in Figure 5 in a quarter of SBZ. For the critical value $J_{N} / J \pm 0.25$ the equation $C=0$ is satisfied only by one in-plane wave vector, corresponding to the high-symmetry point $M$ or $\Gamma$ for FM (Figure 5(a)) or AFM (Figure 5(b)) NNN interactions, respectively. This means that only at this one point does collapse occur and there is no dynamical change of the mode order. For NNN interactions stronger than the critical value solutions of the equation $C=0$ form a line in the SBZ which shifts from the corresponding highsymmetry point toward the center of the SBZ quarter. Thus, as we already mentioned, the region of the reversed mode order appears around the point $M$ for FM NNN interactions and around point $\Gamma$ for AFM ones under the condition that NNN interactions are strong enough.

A similar situation we have for the bcc(110) surface cut; the Hamiltonian matrix is also three-diagonal. In this case the SBZ has the shape of an irregular hexagon which results in rather complicated formulas for the wave vector $\mathbf{k}_{\|}$within the SBZ and, consequently, for the elements of the Hamiltonian matrix. To simplify the description of the problem the socalled uniform zone can be introduced [43], based on new coordinates, $\xi_{1}$ and $\xi_{2}$, assumed to range from -0.5 to 0.5 within the SBZ. With such definition the uniform zone has the shape of a square regardless of the original SBZ geometry. For bcc(110) case the one-to-one correspondence that maps 


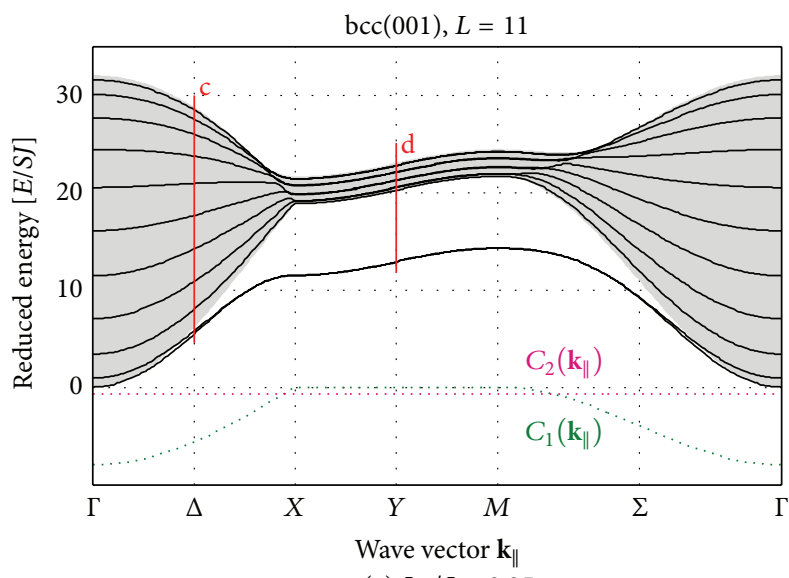

(a) $J_{N} / J=0.35$

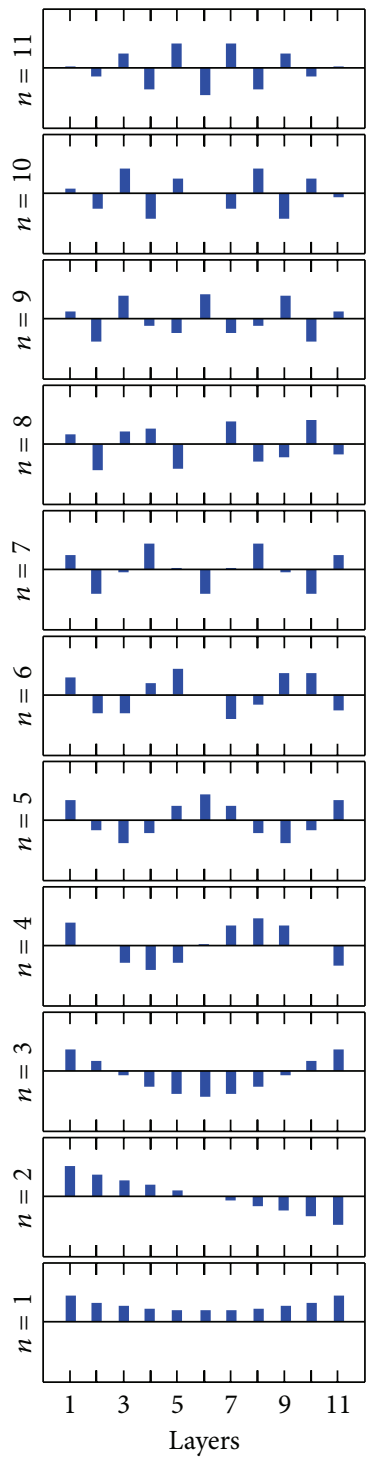

(c)

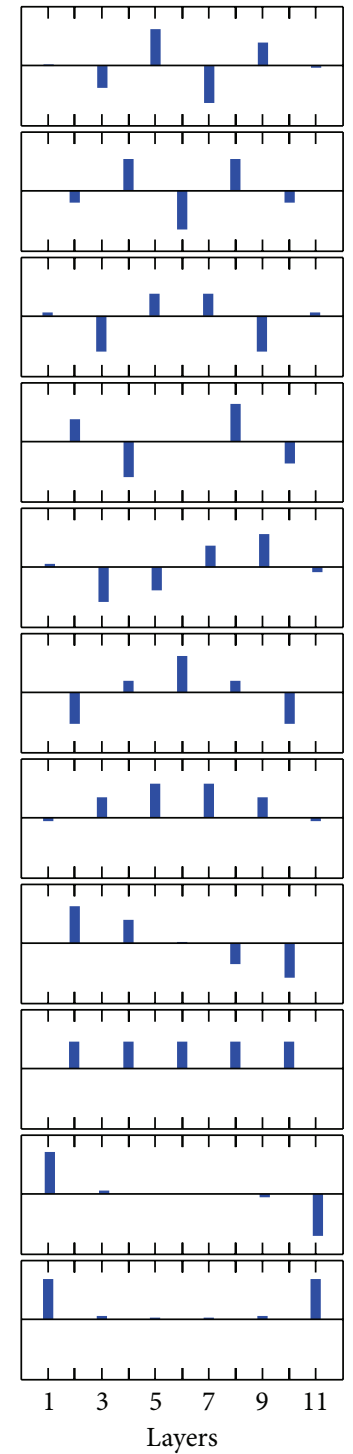

(d)

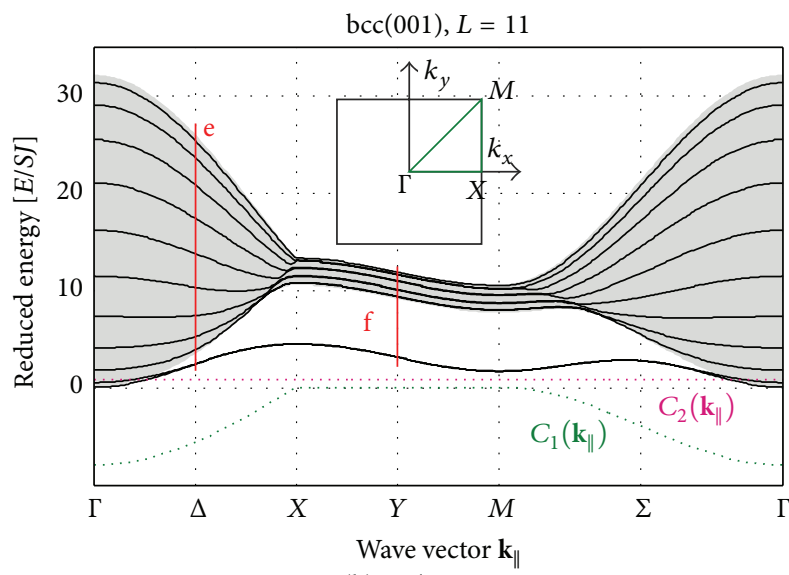

(b) $J_{N} / J=-0.35$

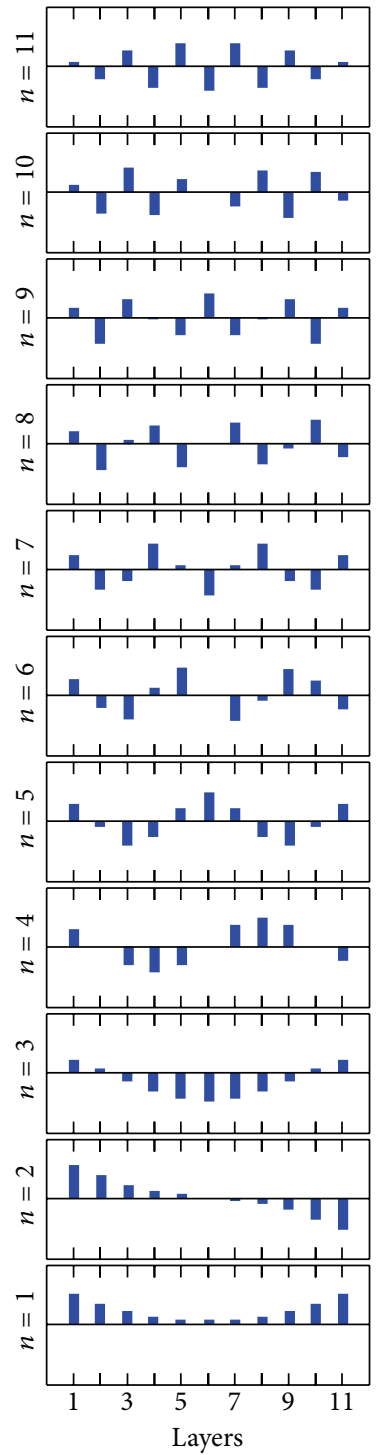

(e)

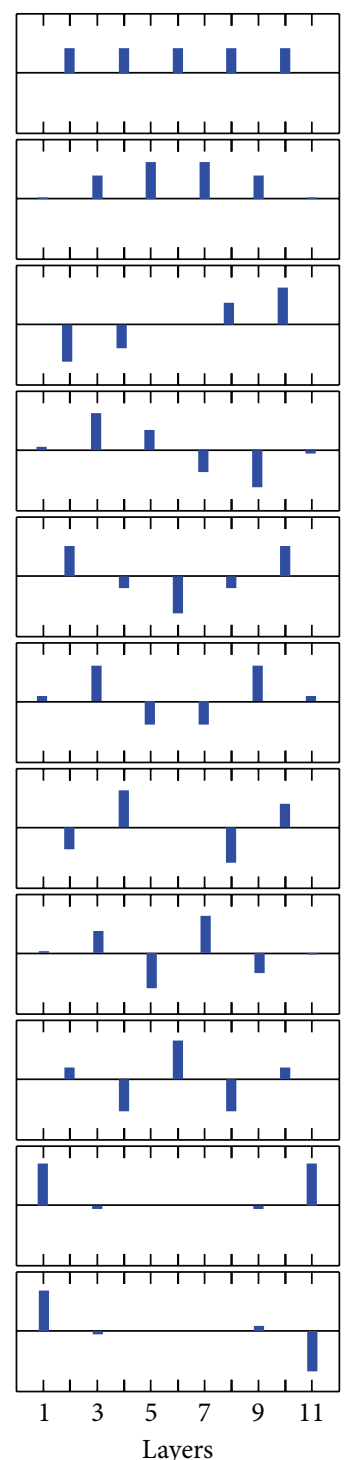

(f)

Figure 8: ((a), (b)) Spin-wave spectra of an 11-layer thick bcc(001) thin film with NNN exchange interactions (a) ferromagnetic and (b) antiferromagnetic. Solid lines represent the energy of individual modes; the shaded area is the bulk band for in-plane spin waves. Each spectrum is plotted along the high-symmetry path shown in (b), inset, and accompanied with the evolution of the off-diagonal Hamiltonian matrix elements $C_{1}$ and $C_{2}$ shown as a green and purple dotted line, respectively. ((c)-(f)) Mode profiles corresponding to points in the SBZ marked with red lines in ((a), (b)). 


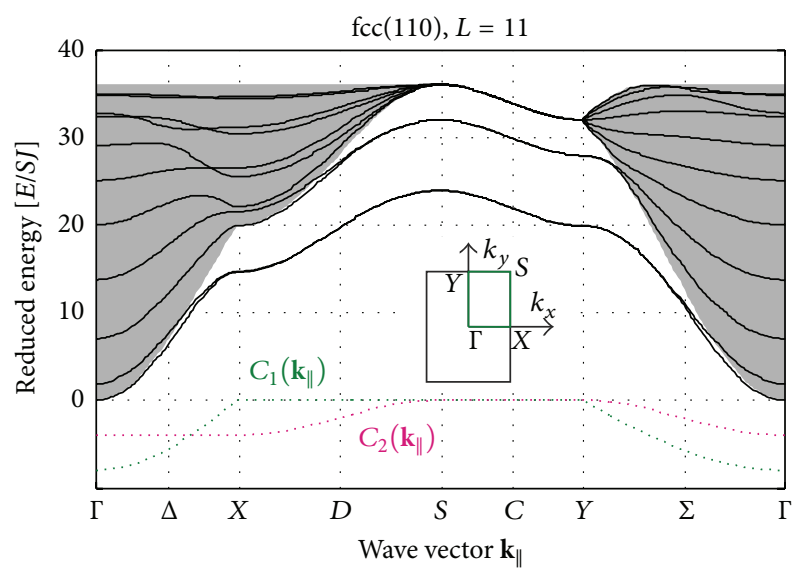

FIGURE 9: Spin-wave spectrum of an 11-layer thick fcc(110) thin film for $J_{N} / J=0.5$, that is, the special case for which the bulk band collapse occurs even for NNN interactions switched on. Solid lines represent the energy of individual modes; the shaded area is the bulk band for in-plane spin waves. The spectrum is plotted along the high-symmetry path shown in (a), inset, and accompanied with the evolution of off-diagonal elements of the Hamiltonian matrix $C_{1}$ (green dotted line) and $C_{2}$ (purple dotted line).

each point in the SBZ to a point in the uniform zone is given by [43]

$$
\mathbf{k}_{\|}\left(\xi_{1}, \xi_{2}\right)=\frac{2 \pi}{a}\left[\sqrt{2} \xi_{1}, 2 \xi_{2}\left(\frac{3}{4}-\left|\xi_{1}\right|\right)\right] .
$$

The off-diagonal element of the Hamiltonian matrix expressed in coordinates $\xi_{1}$ and $\xi_{2}$ is given by

$$
C=-4 S\left(J \cos \pi \xi_{2}+J_{N} \cos \left(2 \pi \xi_{1}+\pi \xi_{2}\right)\right) .
$$

Solutions of the equation $C=0$ for FM NNN interactions are shown in Figure 6. In contrast to sc(001) case there is no threshold of $J_{N} / J$ ratio and the collapse occurs even without NNN interactions. For $J_{N} / J=0$ it happens at the border of the SBZ (segment $Y-D$ ) and is not followed by the mode order reversal. While FM NNN interactions increase the line $C=0$ is moving towards point $X$ and the region of reversed mode order appears. For AFM NNN interactions a necessary condition for the mode order reversal is $J_{N} / J<-1$, which means the NNN interactions must be stronger than $\mathrm{NN}$ ones.

The effect of band collapse followed by mode order reversal should be observable in experiments which gives possibility to look at spin waves over the whole SBZ, for example, by spin polarized electron energy loss spectroscopy (SPEELS) [30]. Observation of this effect gives possibility to determine precisely the $J_{N} / J$ ratio. For example, in $s c(001)$ thin film the eventual collapse appears for some point in between $\Gamma$ and $M$. From relation (9) for the wave vector from the segment $\Gamma-M$ the condition for the collapse is fulfilled if

$$
\frac{J_{N}}{J}=-\frac{1}{4 \cos \left(k_{0} a / \sqrt{2}\right)},
$$

where $k_{0}$ is the length of the wave vector along the $\Gamma-M$ segment for which the collapse occurs and $a$ is the lattice constant.
TABLE 1: The numbers of nearest neighbors (NN) and next nearest (NNN) neighbors in adjacent lattice planes parallel to the surface in cubic thin films and three surface cuts. Individual planes are numbered $l \pm n$, where $n=0, \ldots, 3$ for considered structures.

\begin{tabular}{ccccccccc}
\hline \multirow{2}{*}{ Surface cut } & \multicolumn{5}{c}{ NN } & \multicolumn{6}{c}{ NNN } \\
& $l$ & $l+1$ & $l+2$ & $l+3$ & $l$ & $l+1$ & $l+2$ & $l+3$ \\
\hline sc & & & & & & & & \\
$(001)$ & 4 & 1 & 0 & 0 & 4 & 4 & 0 & 0 \\
$(110)$ & 2 & 2 & 0 & 0 & 2 & 4 & 1 & 0 \\
$(111)$ & 0 & 3 & 0 & 0 & 6 & 0 & 3 & 0 \\
bcc & & & & & & & & \\
$(001)$ & 0 & 4 & 0 & 0 & 4 & 0 & 1 & 0 \\
$(110)$ & 4 & 2 & 0 & 0 & 2 & 2 & 0 & 0 \\
$(111)$ & 0 & 3 & 0 & 1 & 0 & 0 & 3 & 0 \\
fcc & & & & & & & & \\
$(001)$ & 4 & 4 & 0 & 0 & 4 & 0 & 1 & 0 \\
$(110)$ & 2 & 4 & 1 & 0 & 2 & 0 & 2 & 0 \\
$(111)$ & 6 & 3 & 0 & 0 & 0 & 3 & 0 & 0 \\
\hline
\end{tabular}

For bcc(110) surface cut and FM NNN interactions if the wave vector changes along the line $\xi_{1}=0.5\left(1-\xi_{2}\right)$ (the green dashed line in Figure 6) the condition for the collapse is $J_{N} / J=\cos \left(\pi \xi_{2}\right)$. Thus the observation of the collapse for some particular directions in the SBZ gives automatically the ratio of $\mathrm{NNN}$ to $\mathrm{NN}$ interactions.

For other surface cuts the Hamiltonian matrix is threediagonal if NNN interactions are absent and then the collapse occurs only at the border of the SBZ. For example, for sc(110) thin films with only NN interactions taken into account the off-diagonal element of the Hamiltonian matrix is given by

$$
C=-4 S J \cos \frac{1}{\sqrt{2}} k_{x},
$$

which means $C=0$ if $k_{x}= \pm \pi / \sqrt{2}$, which is satisfied by wave vectors from the two borders of the SBZ (bold lines in Figure $7(\mathrm{a}))$. For $\mathrm{fcc}(001)$ surface cut and $J_{N} / J=0$ we have

$$
C=-8 S J \cos \frac{1}{4}\left(k_{x}+k_{y}\right) \cos \frac{1}{4}\left(k_{x}-k_{y}\right),
$$

and thus $C=0$ for the whole SBZ border $\left(k_{x} \pm k_{y}= \pm 2 \pi\right.$, bold lines in Figure $7(b))$. The same situation, that is, the collapse of the bulk band at the SBZ border, exists also for bcc(001) surface cut (see segment $X-M$ in Figure 3(a)) for which

$$
C=-8 S J \cos \frac{1}{2} k_{x} \cos \frac{1}{2} k_{y} .
$$

\section{Separation into Subsystems}

If the Hamiltonian matrix is five-diagonal and the offdiagonal element $C_{1}$ depends on $\mathbf{k}_{\|}$it could happen that $C_{1}=0$ and $C_{2} \neq 0$ for some in-plane wave vectors. In such case, according to the physical meaning of the off-diagonal elements, there is no effective coupling between neighboring layers while next-neighboring layers are coupled. In other words, from the point of view of propagating spin waves the 

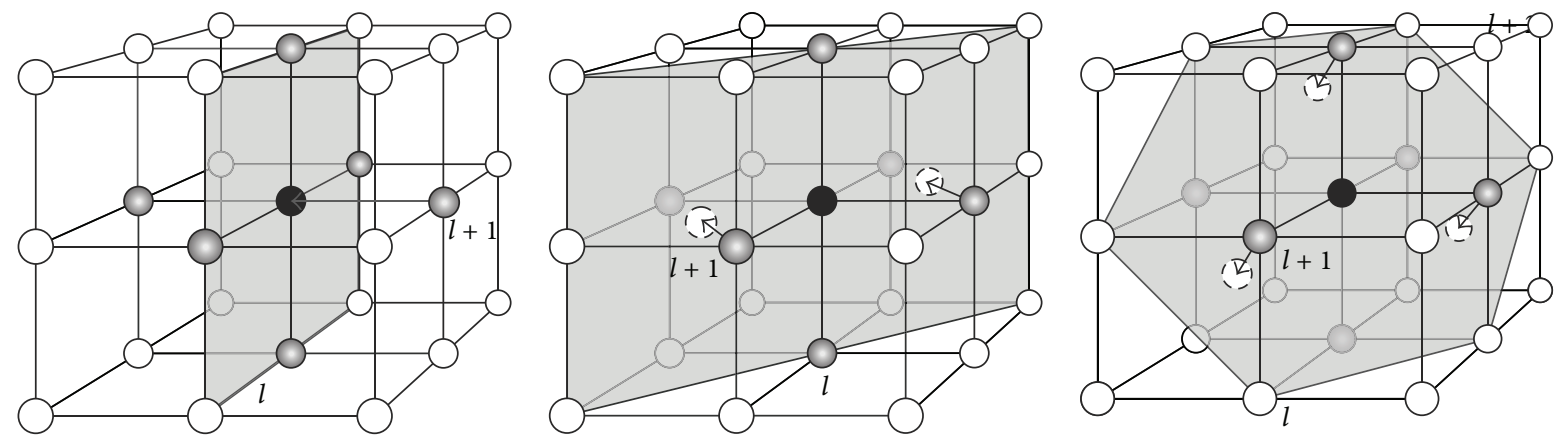

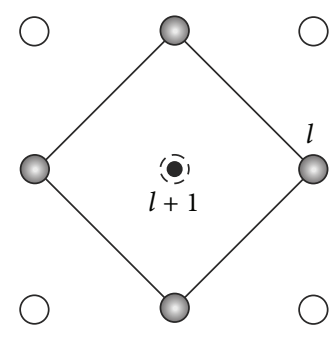

(a) $\operatorname{sc}(001), \mathrm{NN}$
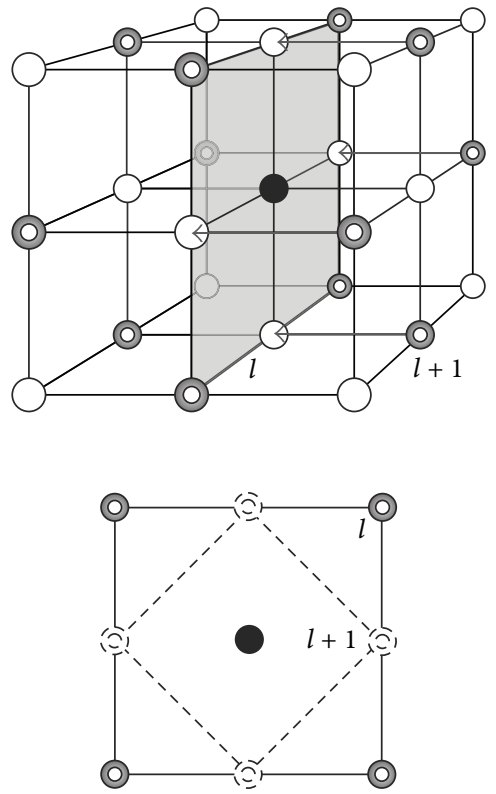

(d) $\operatorname{sc}(001), \mathrm{NNN}$

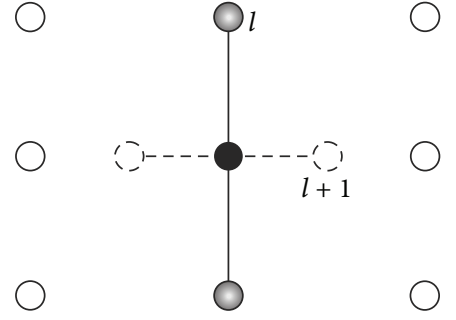

(b) $\mathrm{sc}(110), \mathrm{NN}$
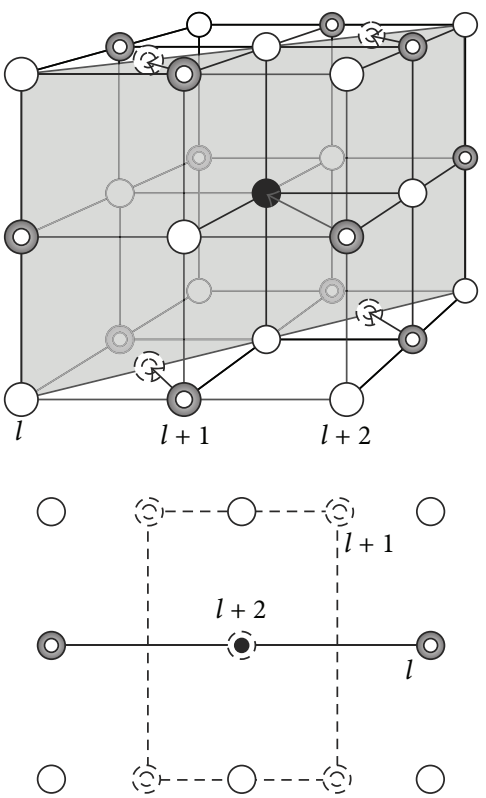

(e) $\operatorname{sc}(110), \mathrm{NNN}$

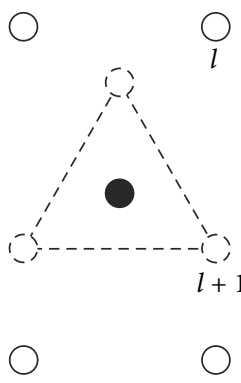

(c) $\operatorname{sc}(111), \mathrm{NN}$
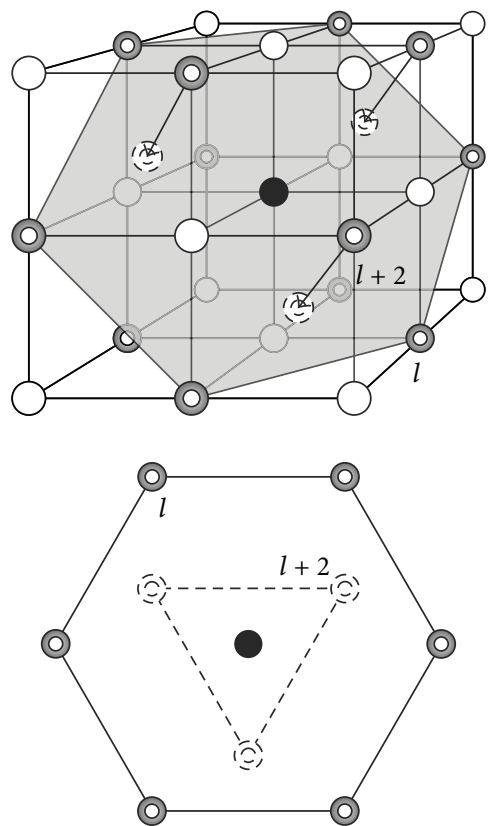

(f) $\mathrm{sc}(111), \mathrm{NNN}$

FIGURE 10: The distribution of ((a)-(c)) nearest, shaded circles, and ((d)-(f)) next nearest, shaded rings, neighbors of an arbitrary chosen spin (the central black circle) in sc thin films for three surface cuts: ((a), (d)) (001), ((b), (e)) (110), and ((c), (f)) (111). (See the text for explanation.)

system is separated into two subsystems consisting of the layers with odd and even numbers, respectively.

In Figure 8 we show the spin-wave spectrum of bcc(001) thin film with FM (panel (a)) and AFM (panel (b)) NNN interactions. For bcc(001) surface cut there are 4 "oblique" $\mathrm{NN}$ at the adjacent layer $(l+1)$ and one "perpendicular" NNN at layer $l+2$. This means that $C_{2}$ depends on NNN interactions only and does not depend on the spin-wave propagation. It has FM character for FM NNN interactions $\left(C_{2}<0\right.$, Figure 8(a)) and AFM character for AFM NNN interactions
$\left(C_{2}>0\right.$, Figure $\left.8(\mathrm{~b})\right)$. Element $C_{1}$ depends only on $\mathrm{NN}$ and $\mathbf{k}_{\|}$; thus in both cases shown in Figure 8 its evolution with $\mathbf{k}_{\|}$is the same: $C_{1}=0$ at the SBZ border, that is, between points $X$ and $M$. For both cases we also show spin-wave profiles (Figures $8(\mathrm{c})-8(\mathrm{f})$ ) for two points in the SBZ: $\Delta$ and $Y$ (marked with red lines in the corresponding spectrum). For point $\Delta$ both spectra are typical for FM thin film but for point $Y$ we can clearly see the separation of the thin film into two subsystems. For two lowest modes, localized at surfaces, the nonzero dynamical magnetization (the amplitude of the spin 

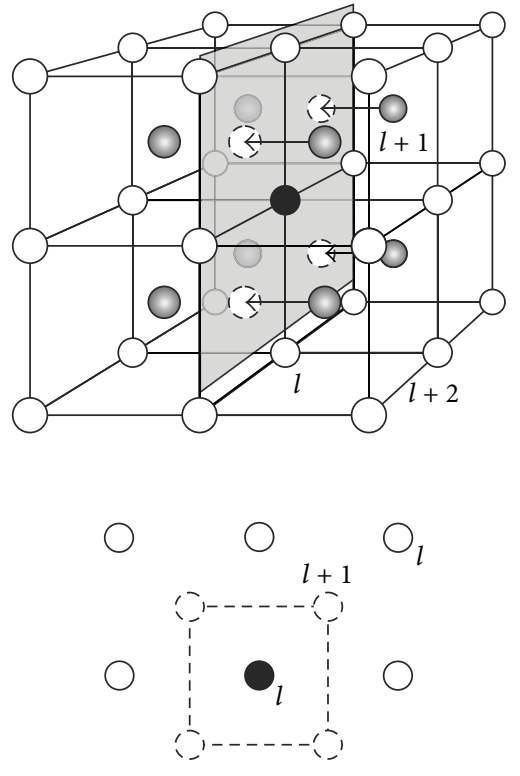

$\bigcirc$

(a) $\mathrm{bcc}(001), \mathrm{NN}$
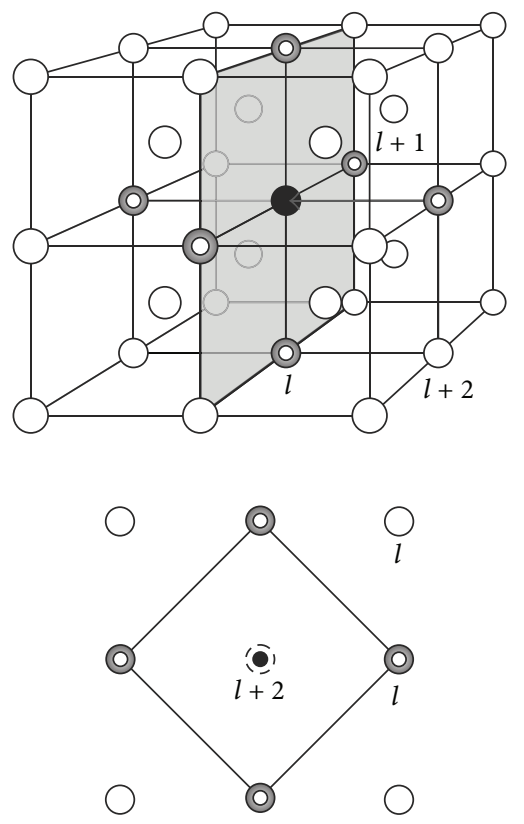

(d) bcc(001), NNN

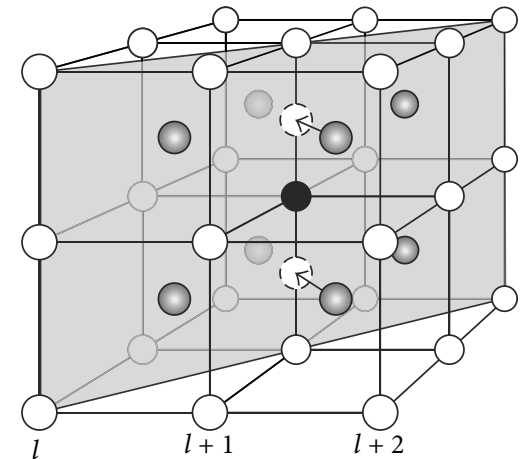

O<smiles>[O]</smiles><smiles>O1O[Al]2O[Al]1O2</smiles>

$\bigcirc$

(b) bcc(110), $\mathrm{NN}$
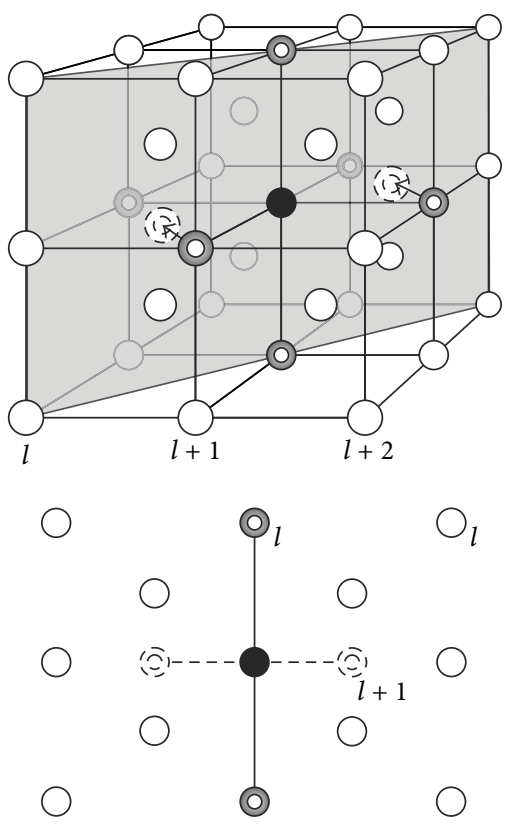

(e) bcc(110), NNN

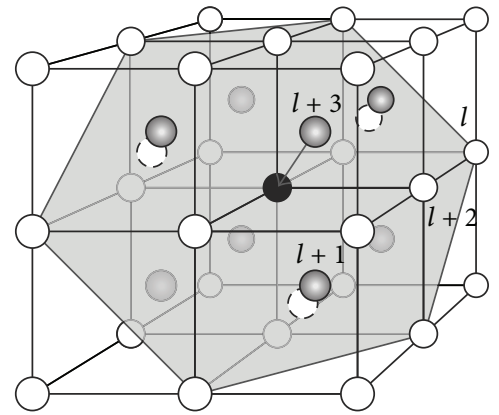

$\bigcirc$

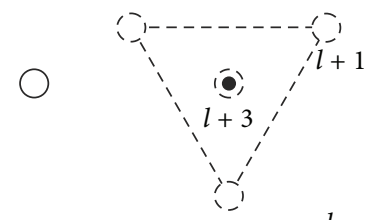

$\stackrel{1}{\circ}$

(c) bcc(111), $\mathrm{NN}$

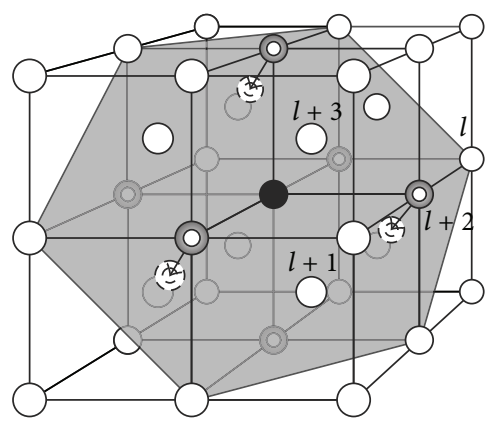

$\bigcirc$
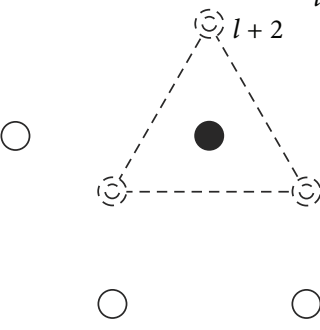

(f) bcc(111), NNN

FIGURE 11: The same distribution and mapping of neighbors as in Figure 10 but for bcc thin films.

precession) occurs only at layers with odd numbers. In the bulk band the spin-wave excitations are restricted to only one type of layers: even- or odd-numbered. For example, the third mode in Figure 8(d) is the uniform mode excited exclusively in the subsystem consisting of only even-numbered layers.

If $C_{1}=0$ the mode order in the spin-wave spectrum is determined by the sign of $C_{2}$ (cf. Section 4). If $C_{2}<0$ the modes appear in normal order in the spectrum (for each subsystem, Figure 8(d)) while for $C_{2}>0$ the mode order is reversed (Figure 8(f)). Since the next-neighboring layer coupling is rather weak this effect is removed if $C_{1} \neq 0$.

If both $C_{1}$ and $C_{2}$ depend on $\mathbf{k}_{\|}$they can vanish simultaneously for some in-plane wave vectors. Such situation occurs for fcc(110) surface cut but only for one value of the $J_{N} / J$ ratio, namely, for 0.5 (Figure 9). In this case the collapse occurs at the SBZ border where both off-diagonal elements $C_{1}$ and $C_{2}$ are equal to zero due to the spin-wave propagation. 

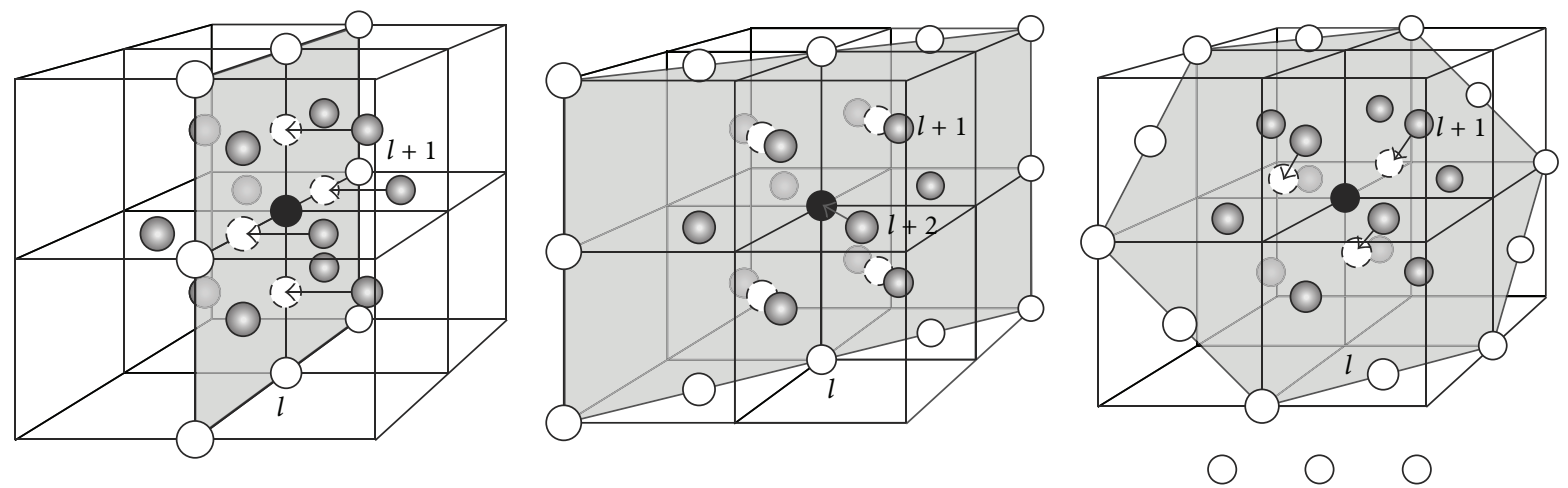

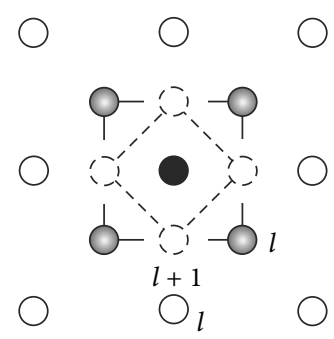

(a) fcc(001), $\mathrm{NN}$
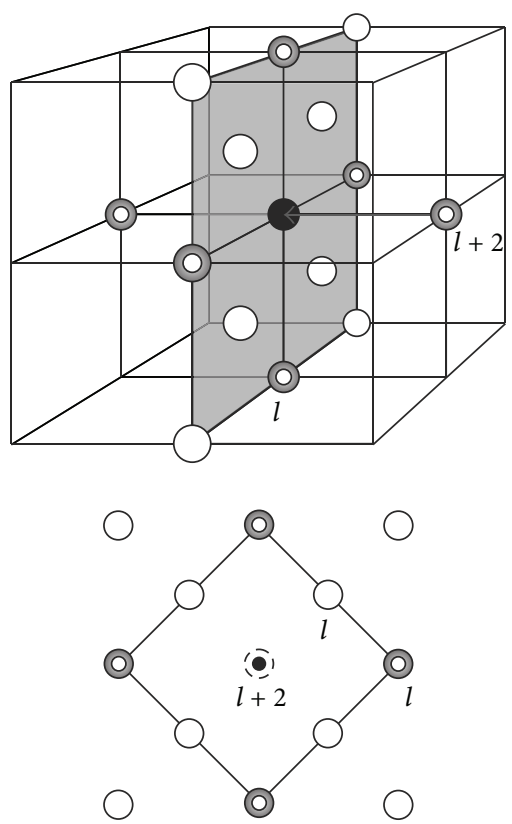

(d) fcc(001), NNN

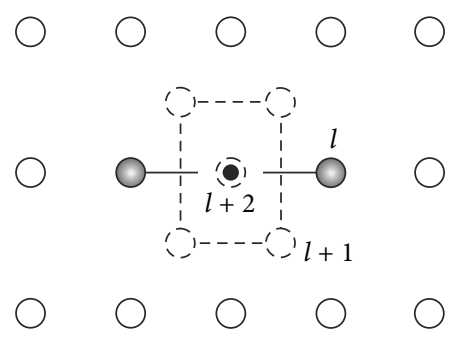

(b) fcc(110), NN
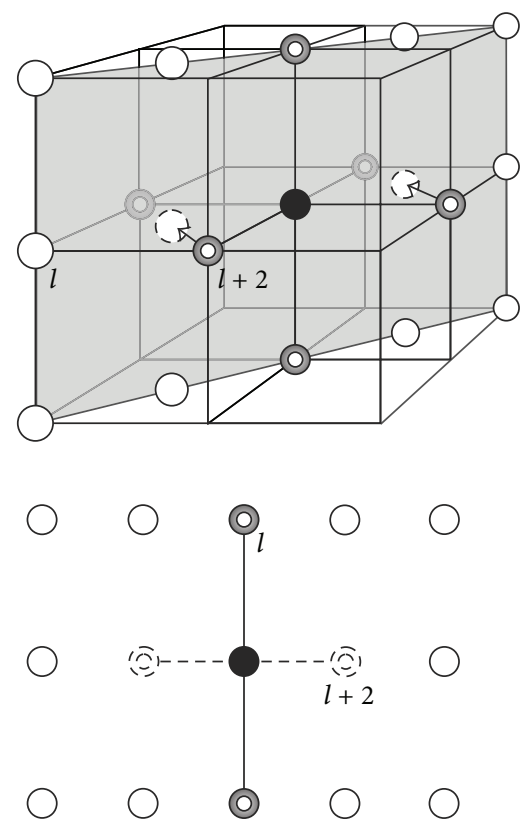

(e) fcc(110), NNN

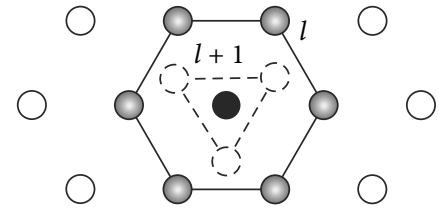

$\bigcirc \bigcirc$

(c) fcc(111), $\mathrm{NN}$
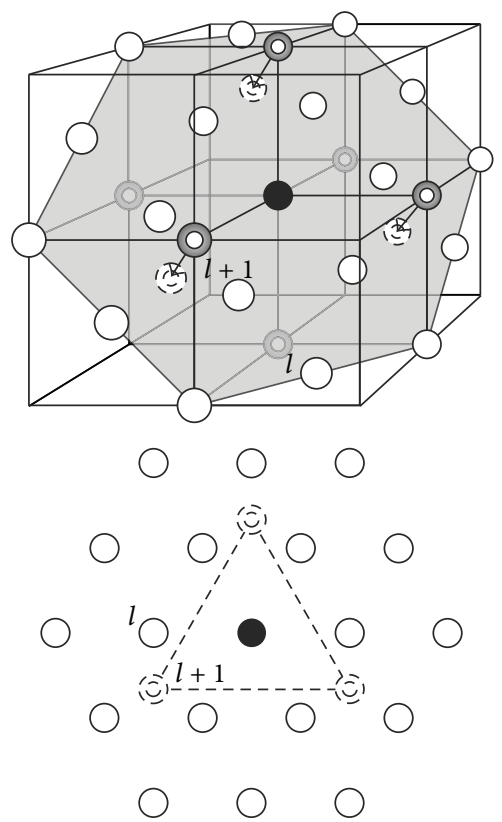

(f) fcc(111), NNN

FIGURE 12: The same distribution and mapping of neighbors as in Figure 10 but for fcc thin films. Due to the large number of lattice sites in this case circles are shown only for spins from the plane $l$ and for respective neighbors.

\section{Conclusion}

In magnetic thin films some interesting phenomena occur just due to the spin-wave propagation. In this paper we collect such dynamic effects in the case of exchange spin waves and link them to properties of Hamiltonian matrix elements. First of all, we study the dynamically induced (due to the wave propagation) surface localization which appears only if neighboring spins are located obliquely with respect to the surface of the film regardless of the circumstance of the interactions. We show that the same rule holds for the subsurface localization as well.

Another effect is the collapse of the bulk band into one energy level for some certain wave vectors caused by the vanishing of the effective coupling between adjacent layers of spins. The collapse, usually followed by the reversal of the mode order in the spin-wave spectrum, can be utilized for precise determination of the next nearest neighbors exchange interactions. If the effective interlayer coupling ranges up to the next-neighboring layers the spin waves from the border of 
TABLE 2: Structural sums $\Gamma_{n}$ for nearest neighbors disposed in adjacent lattice planes $l \pm n, n=0, \ldots, 3$, parallel to the surface in thin films considered in the paper.

\begin{tabular}{|c|c|c|c|c|}
\hline Surface cut & $\Gamma_{0}$ & $\Gamma_{1}$ & $\Gamma_{2}$ & $\Gamma_{3}$ \\
\hline \multicolumn{5}{|l|}{ sc } \\
\hline$(001)$ & $2 \cos \left(k_{1}\right)+2 \cos \left(k_{2}\right)$ & 1 & 0 & 0 \\
\hline (110) & $2 \cos \left(k_{2}\right)$ & $2 \cos \left(\frac{k_{1}}{2}\right)$ & 0 & 0 \\
\hline (111) & 0 & $\exp \left(i \frac{2 k_{2}}{3}\right)+2 \exp \left(-i \frac{k_{2}}{3}\right) \cos (\alpha)$ & 0 & 0 \\
\hline \multicolumn{5}{|c|}{ ( } \\
\hline$(001)$ & 0 & $4 \cos \left(\frac{k_{1}}{2}\right) \cos \left(\frac{k_{2}}{2}\right)$ & 0 & 0 \\
\hline (110) & $4 \cos \left(k_{1}\right) \cos \left(\left(\frac{3}{4}-\frac{\left|k_{1}\right|}{2 \pi}\right) k_{2}\right)$ & $2 \cos \left(\left(\frac{3}{4}-\frac{\left|k_{1}\right|}{2 \pi}\right) k_{2}\right)$ & 0 & 0 \\
\hline (111) & 0 & $\exp \left(-i \frac{2 k_{2}}{3}\right)+2 \exp \left(i \frac{k_{2}}{3}\right) \cos (\alpha)$ & 0 & 1 \\
\hline \multicolumn{5}{|c|}{ 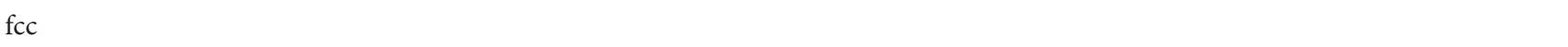 } \\
\hline$(001)$ & $2 \cos \left(k_{1}\right)+2 \cos \left(k_{2}\right)$ & $4 \cos \left(\frac{k_{1}}{2}\right) \cos \left(\frac{k_{2}}{2}\right)$ & 0 & 0 \\
\hline (110) & $2 \cos \left(k_{2}\right)$ & $4 \cos \left(\frac{k_{1}}{2}\right) \cos \left(\frac{k_{2}}{2}\right)$ & 1 & 0 \\
\hline (111) & $4 \cos \left(k_{2}\right) \cos (\alpha)+4 \cos ^{2}(\alpha)-2$ & $\exp \left(-i \frac{2 k_{2}}{3}\right)+2 \exp \left(-i \frac{k_{2}}{3}\right) \cos (\alpha)$ & 0 & 0 \\
\hline
\end{tabular}

Symbol: $\alpha=\left(k_{1} / 3\right)\left(2-\left|k_{2}\right| / \pi\right)$.

TABLE 3: Structural sums $\Gamma_{n}^{N}$ for next nearest neighbors disposed in adjacent lattice planes $l \pm n, n=0, \ldots, 2$, parallel to the surface in thin films considered in the paper.

\begin{tabular}{|c|c|c|c|}
\hline Surface cut & $\Gamma_{0}^{N}$ & $\Gamma_{1}^{N}$ & $\Gamma_{2}^{N}$ \\
\hline \multicolumn{4}{|l|}{ sc } \\
\hline$(001)$ & $4 \cos \left(k_{1}\right) \cos \left(k_{2}\right)$ & $2 \cos \left(k_{1}\right)+2 \cos \left(k_{2}\right)$ & 0 \\
\hline (110) & $2 \cos \left(k_{1}\right)$ & $4 \cos \left(\frac{k_{1}}{2}\right) \cos \left(k_{2}\right)$ & 1 \\
\hline (111) & $4 \cos \left(k_{2}\right) \cos (\alpha)+4 \cos ^{2}(\alpha)-2$ & 0 & $\exp \left(-i \frac{2 k_{2}}{3}\right)+2 \exp \left(i \frac{k_{2}}{3}\right) \cos (\alpha)$ \\
\hline \multicolumn{4}{|l|}{ bcc } \\
\hline$(001)$ & $2 \cos \left(k_{1}\right)+2 \cos \left(k_{2}\right)$ & 0 & 1 \\
\hline$(110)$ & $2 \cos \left(\left(\frac{3}{2}-\frac{\left|k_{1}\right|}{\pi}\right) k_{2}\right)$ & $2 \cos \left(k_{1}\right)$ & 0 \\
\hline (111) & 0 & 0 & $\exp \left(i \frac{2 k_{2}}{3}\right)+2 \exp \left(-i \frac{k_{2}}{3}\right) \cos (\alpha)$ \\
\hline \multicolumn{4}{|l|}{ fcc } \\
\hline$(001)$ & $4 \cos \left(k_{1}\right) \cos \left(k_{2}\right)$ & 0 & 1 \\
\hline$(110)$ & $2 \cos \left(k_{1}\right)$ & 0 & $2 \cos \left(k_{2}\right)$ \\
\hline (111) & 0 & $\exp \left(i \frac{4 k_{2}}{3}\right)+2 \exp \left(-i \frac{2 k_{2}}{3}\right) \cos (2 \alpha)$ & 0 \\
\hline
\end{tabular}

Symbol: $\alpha=\left(k_{1} / 3\right)\left(2-\left|k_{2}\right| / \pi\right)$. 
the surface Brillouin zone may exhibit another phenomenon: they can be excited in only every second lattice plane; that is, the thin film acts like that divided into two subsystems: one consists of odd-numbered layers and the second one consists of even-numbered ones.

\section{Appendices}

\section{A. Nearest and Next Nearest Neighbors Distribution}

Here we present an analysis of the nearest (NN) and next nearest (NNN) neighbors distribution in thin films discussed in this paper. In Figures 10-12 the 3D distribution is provided as well as $2 \mathrm{D}$ mapping onto the lattice plane parallel to the surface of the film. In each figure an arbitrary chosen spin is marked as the central black circle and the shaded plane represents the plane $l$ parallel to the film surface which contains the chosen spin. Below each 3D distribution the $2 \mathrm{D}$ mapping of respective neighbors is shown, that is, their projection onto the plane $l$, where shaded circles (rings) represent $\mathrm{NN}(\mathrm{NNN})$ from the same plane $l$ while empty dashed ones represent the projections of respective neighbors from adjacent planes $(l+n)$.

The numbers of neighbors within the individual lattice planes parallel to the surface are summarized in Table 1.

\section{B. Structural Sums}

In this appendix we provide the explicit dependence on the in-plane wave vector $\mathbf{k}_{\|}=\left(k_{x}, k_{y}\right)$ of structural sums for thin films considered in the paper for NN (Table 2) and NNN (Table 3). For the detailed calculations please see [67].

\section{Conflict of Interests}

The author declares that there is no conflict of interests regarding the publication of this paper.

\section{Acknowledgments}

The author thanks Jean-Claude Serge Lévy for valuable discussions. This study received support from the National Science Centre (NCN) of Poland, Project DEC-2012/07/E/ST3/ 00538. The author acknowledges the financial support of the Operational Programme Human Capital, cofinanced by the European Social Fund in Poland, Grant no. POKL.04.01.0100-019/10.

\section{References}

[1] M. J. Klein and R. S. Smith, "Thin ferromagnetic films," Physical Review, vol. 81, no. 3, pp. 378-380, 1951.

[2] P. E. Wigen, C. F. Kooi, M. R. Shanabarger, and T. D. Rossing, "Dynamic pinning in thin-film spin-wave resonance," Physical Review Letters, vol. 9, no. 5, pp. 206-208, 1962.

[3] P. Grünberg, J. Barnas, F. Saurenbach, J. A. Fuss, A. Wolf, and M. Vohl, "Layered magnetic structures: antiferromagnetic type interlayer coupling and magnetoresistance due to antiparallel alignment," Journal of Magnetism and Magnetic Materials, vol. 93, pp. 58-66, 1991.

[4] J. Dubowik, K. Załęski, H. Głowiński, and I. Gociańska, “Angular dependence of ferromagnetic resonance linewidth in thin films," Physical Review B, vol. 84, no. 18, Article ID 184438, 2011.

[5] P. Kuświk, P. L. Gastelois, M. M. Soares et al., "Effect of $\mathrm{CoO} / \mathrm{Ni}$ orthogonal exchange coupling on perpendicular anisotropy of Ni films on Pd(001)," Physical Review B, vol. 91, no. 13, Article ID 134413, 2015.

[6] A. R. Ferchmin, "A quantum theory of spin-wave resonance in cubic ferromagnets," Physics Letters, vol. 1, no. 7, pp. 281-283, 1962.

[7] H. Puszkarski, "Theory of surface states in spin wave resonance," Progress in Surface Science, vol. 9, no. 5-6, pp. 191-247, 1979.

[8] I. Zasada, B. Busiakiewicz, and L. Wojtczak, "Short-range order in Valenta model of thin films," Journal of Magnetism and Magnetic Materials, vol. 312, no. 1, pp. 58-64, 2007.

[9] D.-T. Hoang, M. Kasperski, H. Puszkarski, and H. T. Diep, "Reorientation transition in molecular thin films: potts model with dipolar interaction," Journal of Physics Condensed Matter, vol. 25, no. 5, Article ID 056006, 2013.

[10] H. Puszkarski and P. Tomczak, "Spin-wave resonance in $(\mathrm{Ga}, \mathrm{Mn})$ As thin films: probing in-plane surface magnetic anisotropy," Physical Review B, vol. 91, no. 19, Article ID 195437, 2015.

[11] C. A. F. Vaz, J. A. C. Bland, and G. Lauhoff, "Magnetism in ultrathin film structures," Reports on Progress in Physics, vol. 71, no. 5, Article ID 056501, 2008.

[12] M. Krawczyk, H. Puszkarski, J.-C. S. Lévy, S. Mamica, and D. Mercier, "Theoretical study of spin wave resonance filling fraction effect in composite ferromagnetic [A-B-A] trilayer," Journal of Magnetism and Magnetic Materials, vol. 246, no. 1-2, pp. 93-100, 2002.

[13] W. P. Zhou and G. H. Yun, "The energy bands and the dispersion relations of the spin waves in a ferromagnetic bilayer system," Surface Science, vol. 553, no. 1-3, pp. 75-81, 2004.

[14] S. Pal, B. Rana, S. Saha et al., "Time-resolved measurement of spin-wave spectra in $\mathrm{CoO}$ capped $[\mathrm{Co}(t) / \mathrm{Pt}(7 \AA)]_{n-1} \mathrm{Co}(t)$ multilayer systems," Journal of Applied Physics, vol. 111, no. 7, Article ID 07C507, 2012.

[15] V. V. Kruglyak, P. S. Keatley, A. Neudert, R. J. Hicken, J. R. Childress, and J. A. Katine, "Imaging collective magnonic modes in 2D arrays of magnetic nanoelements," Physical Review Letters, vol. 104, no. 2, Article ID 027201, 2010.

[16] S. Tacchi, G. Duerr, J. W. Klos et al., "Forbidden band gaps in the spin-wave spectrum of a two-dimensional bicomponent magnonic crystal," Physical Review Letters, vol. 109, no. 13, Article ID 137202, 2012.

[17] S. Mamica, M. Krawczyk, and J. W. Kos, "Spin-wave band structure in 2D magnonic crystals with elliptically shaped scattering centres," Advances in Condensed Matter Physics, vol. 2012, Article ID 161387, 6 pages, 2012.

[18] M. L. Sokolovskyy, J. W. Klos, S. Mamica, and M. Krawczyk, "Calculation of the spin-wave spectra in planar magnonic crystals with metallic overlayers," Journal of Applied Physics, vol. 111, no. 7, Article ID 07C515, 2012.

[19] R. W. Damon and J. R. Eshbach, "Magnetostatic modes of a ferromagnet slab," Journal of Physics and Chemistry of Solids, vol. 19, no. 3-4, pp. 308-320, 1961. 
[20] M. Mruczkiewicz, M. Krawczyk, G. Gubbiotti et al., "Nonreciprocity of spin waves in metallized magnonic crystal," New Journal of Physics, vol. 15, Article ID 113023, 2013.

[21] H. Puszkarski, J. C. S. Lévy, and S. Mamica, "Does the generation of surface spin-waves hinge critically on the range of neighbour interaction?" Physics Letters A, vol. 246, no. 3-4, pp. 347-352, 1998.

[22] S. Mamica, R. Józefowicz, and H. Puszkarski, "The role of oblique-to-surface disposition of neighbours in the emergence of surface spin waves in magnetic films," Acta Physica Polonica A, vol. 94, no. 1, pp. 79-91, 1998.

[23] J. C. Lévy, E. Ilisca, and J. L. Motchane, "Influence of surface anisotropy and next-nearest-neighbor coupling on surface spin waves," Physical Review B, vol. 5, no. 3, pp. 1099-1105, 1972.

[24] H. Puszkarski, "Surface spin waves in hexagonal cobalt thin films," Physica Status Solidi (B), vol. 22, no. 2, pp. 355-359, 1967.

[25] J. C. S. Lévy, "Surface and interface magnons: magnetic structures near the surface," Surface Science Reports, vol. 1, no. 2, pp. 39-119, 1981.

[26] B. X. Xu, M. Mostoller, and A. K. Rajagopal, "Spin waves at surfaces and interfaces in cubic Heisenberg systems," Physical Review B, vol. 31, no. 11, pp. 7413-7423, 1985.

[27] J. M. Wesselinowa, "Influence of magnetic surface anisotropy on the dynamic properties in ferromagnetic thin films," Journal of Physics Condensed Matter, vol. 18, no. 35, pp. 8169-8178, 2006.

[28] M. Plihal, D. L. Mills, and J. Kirschner, "Spin wave signature in the spin polarized electron energy loss spectrum of ultrathin $\mathrm{Fe}$ films: theory and experiment," Physical Review Letters, vol. 82, no. 12, pp. 2579-2582, 1999.

[29] H. Ibach, D. Bruchmann, R. Vollmer, M. Etzkorn, P. S. A. Kumar, and J. Kirschner, "A novel spectrometer for spinpolarized electron energy-loss spectroscopy," Review of Scientific Instruments, vol. 74, no. 9, pp. 4089-4095, 2003.

[30] R. Vollmer, M. Etzkorn, P. S. Anil Kumar, H. Ibach, and J. Kirschner, "Spin-polarized electron energy loss spectroscopy of high energy, large wave vector spin waves in ultrathin fcc Co films on $\mathrm{Cu}(001), "$ Physical Review Letters, vol. 91, no. 14, p. 147201/4, 2003.

[31] K. Zakeri, T.-H. Chuang, A. Ernst et al., "Direct probing of the exchange interaction at buried interfaces," Nature Nanotechnology, vol. 8, no. 11, pp. 853-858, 2013.

[32] T.-H. Chuang, K. Zakeri, A. Ernst et al., "Magnetic properties and magnon excitations in $\mathrm{Fe}(001)$ films grown on $\operatorname{Ir}(001)$, Physical Review B, vol. 89, no. 17, Article ID 174404, 2014.

[33] K. Zakeri, J. Prokop, Y. Zhang, and J. Kirschner, "Magnetic excitations in ultrathin magnetic films: temperature effects," Surface Science, vol. 630, pp. 311-316, 2014.

[34] Y. Meng, Kh. Zakeri, A. Ernst et al., "Direct evidence of antiferromagnetic exchange interaction in $\mathrm{Fe}(001)$ films: strong magnon softening at the high-symmetry M point," Physical Review B, vol. 90, no. 17, Article ID 174437, 2014.

[35] K. Zakeri, Y. Zhang, J. Prokop et al., "Asymmetric spin-wave dispersion on $\mathrm{Fe}(110)$ : direct evidence of the dzyaloshinskiimoriya interaction," Physical Review Letters, vol. 104, no. 13, Article ID 137203, 2010.

[36] K. Zakeri, Y. Zhang, and J. Kirschner, "Surface magnons probed by spin-polarized electron energy loss spectroscopy," Journal of Electron Spectroscopy and Related Phenomena, vol. 189, pp. 157163, 2013.

[37] P. Grünberg, "Magnetostatic spinwave modes of a heterogeneous ferromagnetic double layer," Journal of Applied Physics, vol. 52, no. 11, pp. 6824-6829, 1981.
[38] J. Barnaś, "Interlayer exchange coupling in ultra-thin layered structures," Journal of Magnetism and Magnetic Materials, vol. 128, no. 1-2, pp. 171-178, 1993.

[39] R. W. Wang and D. L. Mills, "Magnetic properties of finite antiferromagnetic superlattices: statics, dynamics, and the surface spin-flop phase," Physical Review B, vol. 50, no. 6, pp. 3931-3941, 1994.

[40] S. M. Rezende, C. Chesman, M. A. Lucena, A. Azevedo, F. M. De Aguiar, and S. S. P. Parkin, "Studies of coupled metallic magnetic thin-film trilayers," Journal of Applied Physics, vol. 84, no. 2, pp. 958-972, 1998.

[41] R. Zivieri, L. Giovannini, and F. Nizzoli, "Acoustical and optical spin modes of multilayers with ferromagnetic and antiferromagnetic coupling," Physical Review B, vol. 62, no. 22, Article ID 14950, 2000.

[42] R. Zivieri, "Metamaterial properties of one-dimensional and two-dimensional magnonic crystals," Solid State Physics, vol. 63, pp. 151-216, 2012.

[43] S. Mamica, "Dynamic effects on the spin-wave spectrum of the bcc thin film," The European Physical Journal B, vol. 87, no. 12, 2014.

[44] E. Y. Vedmedenko, A. Ghazali, and J.-C. S. Lévy, "Magnetic structures of Ising and vector spins monolayers by Monte-Carlo simulations," Surface Science, vol. 402-404, pp. 391-395, 1998.

[45] T. Shinjo, T. Okuno, R. Hassdorf, K. Shigeto, and T. Ono, "Magnetic vortex core observation in circular dots of permalloy," Science, vol. 289, no. 5481, pp. 930-932, 2000.

[46] K. L. Metlov and Y. P. Lee, "Map of metastable states for thin circular magnetic nanocylinders," Applied Physics Letters, vol. 92, no. 11, Article ID 112506, 2008.

[47] S. Mamica, J.-C. S. Lévy, P. Depondt, and M. Krawczyk, “The effect of the single-spin defect on the stability of the in-plane vortex state in 2D magnetic nanodots," Journal of Nanoparticle Research, vol. 13, no. 11, pp. 6075-6083, 2011.

[48] S. Mamica, J.-C. S. Lévy, M. Krawczyk, and P. Depondt, "Stability of the Landau state in square two-dimensional magnetic nanorings," Journal of Applied Physics, vol. 112, no. 4, Article ID 043901, 2012.

[49] L. Rondin, J.-P. Tetienne, S. Rohart et al., "Stray-field imaging of magnetic vortices with a single diamond spin," Nature Communications, vol. 4, article 2279, 2013.

[50] X. Li, W. V. Liu, and L. Balents, "Spirals and skyrmions in two dimensional oxide heterostructures," Physical Review Letters, vol. 112, no. 6, Article ID 067202, 2014.

[51] O. G. Heinonen, D. K. Schreiber, and A. K. Petford-Long, "Micromagnetic modeling of spin-wave dynamics in exchangebiased permalloy disks," Physical Review B, vol. 76, no. 14, Article ID 144407, 2007.

[52] L. Giovannini, F. Montoncello, R. Zivieri, and F. Nizzoli, "Spin excitations in nanometric magnetic dots: calculations and comparison with light scattering measurements," Journal of Physics Condensed Matter, vol. 19, no. 22, Article ID 225008, 2007.

[53] Z. Liu, R. D. Sydora, and M. R. Freeman, "Shape effects on magnetization state transitions in individual 160-nm diameter Permalloy disks," Physical Review B, vol. 77, no. 17, Article ID 174410, 2008.

[54] F. Montoncello, L. Giovannini, F. Nizzoli, R. Zivieri, G. Consolo, and G. Gubbiotti, "Spin-wave activation by spin-polarized current pulse in magnetic nanopillars," Journal of Magnetism and Magnetic Materials, vol. 322, no. 16, pp. 2330-2334, 2010. 
[55] S. Mamica, J.-C. S. Lévy, and M. Krawczyk, "Effects of the competition between the exchange and dipolar interactions in the spin-wave spectrum of two-dimensional circularly magnetized nanodots," Journal of Physics D: Applied Physics, vol. 47, no. 1, Article ID 015003, 2014.

[56] S. Mamica, M. Krawczyk, M. L. Sokolovskyy, and J. RomeroVivas, "Large magnonic band gaps and spectra evolution in three-dimensional magnonic crystals based on magnetoferritin nanoparticles," Physical Review B, vol. 86, no. 14, Article ID 144402, 2012.

[57] V. S. Tkachenko, A. N. Kuchko, M. Dvornik, and V. V. Kruglyak, "Propagation and scattering of spin waves in curved magnonic waveguides," Applied Physics Letters, vol. 101, no. 15, Article ID 152402, 2012.

[58] M. Krawczyk, S. Mamica, M. Mruczkiewicz et al., "Magnonic band structures in two-dimensional bi-component magnonic crystals with in-plane magnetization," Journal of Physics D: Applied Physics, vol. 46, no. 49, Article ID 495003, 2013.

[59] S. Mamica, H. Puszkarski, and J. C. S. Lévy, "The role of nextnearest neighbours for the existence conditions of subsurface spin waves in magnetic films," Physica Status Solidi B, vol. 218, no. 2, pp. 561-569, 2000.

[60] S. Mühlbauer, P. Böni, R. Georgii, A. Schmehl, D. G. Schlom, and J. Mannhart, "Field and temperature dependence of the magnetization in ferromagnetic EuO thin films," Journal of Physics Condensed Matter, vol. 20, no. 10, Article ID 104230, 2008.

[61] W. Söllinger, W. Heiss, R. T. Lechner et al., "Exchange interactions in europium monochalcogenide magnetic semiconductors and their dependence on hydrostatic strain," Physical Review B-Condensed Matter and Materials Physics, vol. 81, no. 15, Article ID 155213, 2010.

[62] P. Liu, J. A. C. Santana, Q. Dai, X. Wang, P. A. Dowben, and J. Tang, "Sign of the superexchange coupling between next-nearest neighbors in EuO," Physical Review B-Condensed Matter and Materials Physics, vol. 86, no. 22, Article ID 224408, 2012.

[63] R. F. Wallis, A. A. Maradudin, I. P. Ipatova, and A. A. Klochikhin, "Surface spin waves," Solid State Communications, vol. 5, no. 1, pp. 89-92, 1967.

[64] H. Puszkarski and S. Mamica, "Interface-localized mode in bilayer film ferromagnetic resonance spectrum," Horizons in World Physics, vol. 244, pp. 1-43, 2004.

[65] C. Kittel, Introduction to Solid State Physics, Wiley, 2004.

[66] S. Mamica, "Effects of spin-wave energy degeneracy induced by in-plane wave propagation in ferromagnetic thin films," Journal of Magnetism and Magnetic Materials, vol. 322, no. 20, pp. 29942999, 2010.

[67] S. Mamica and H. Puszkarski, "Nearest and next-nearest neighbourhood surface mapping in thin cubic films. Properties of structural sums," Acta Physicae Superficierum, vol. 9, no. 9, pp. 17-70, 2006. 

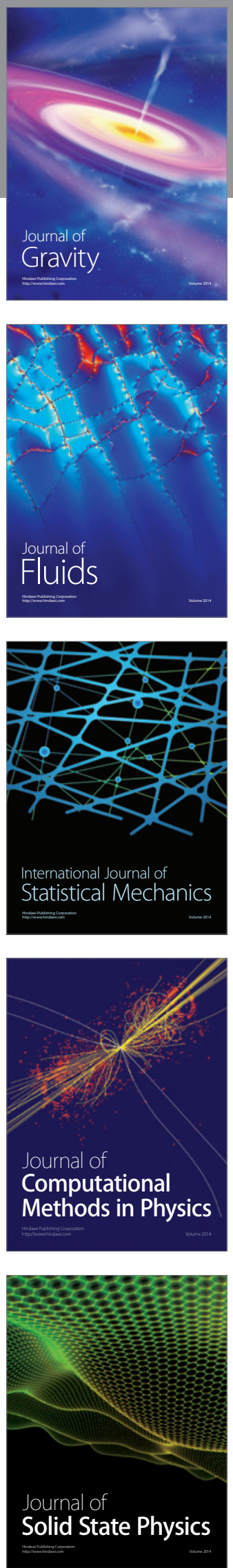

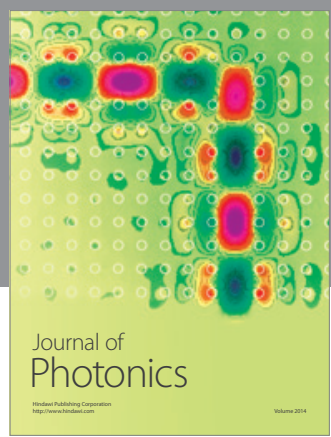

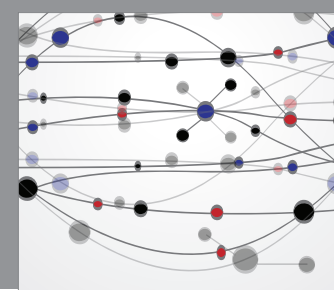

The Scientific World Journal

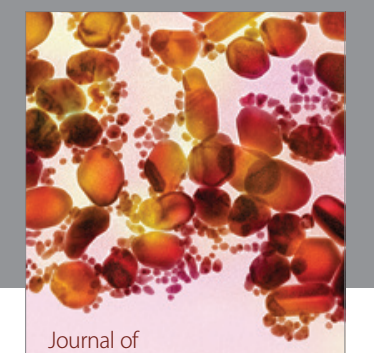

Soft Matter
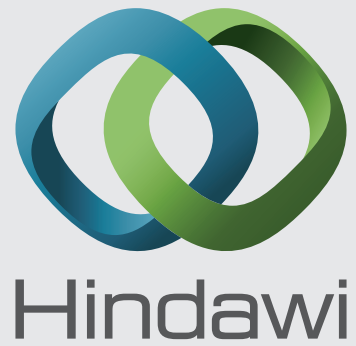

Submit your manuscripts at

http://www.hindawi.com
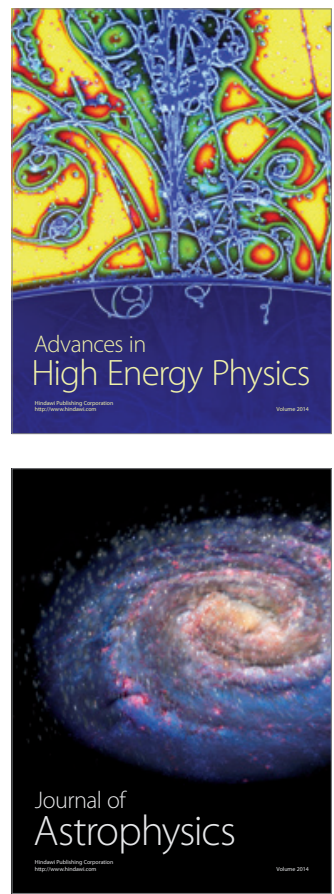
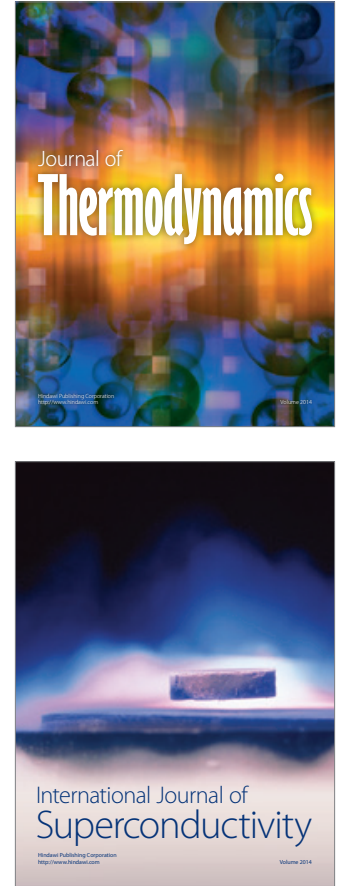
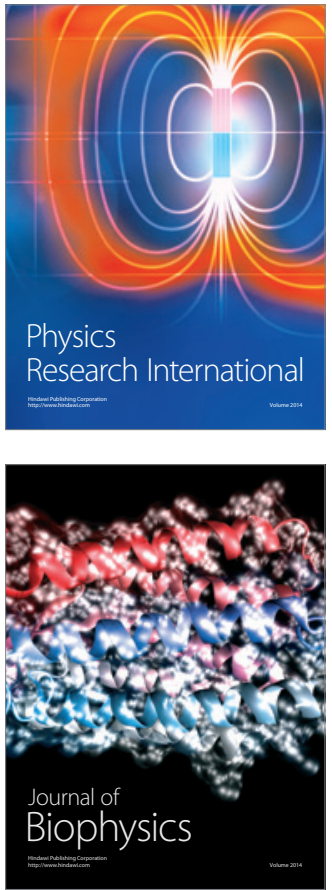
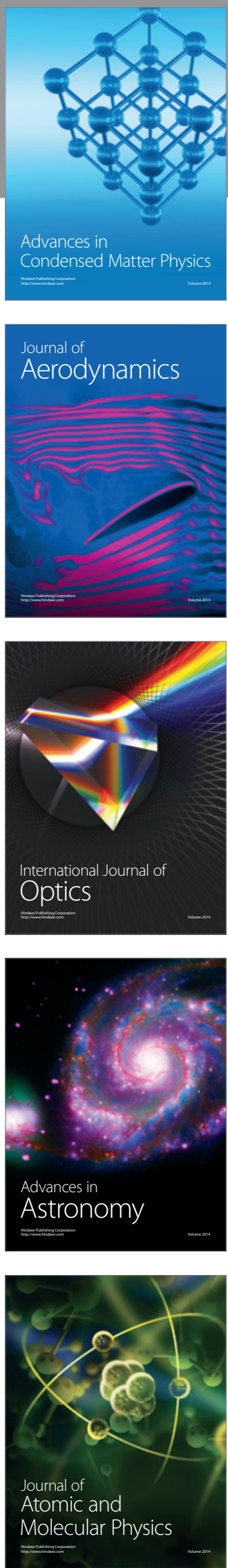